\title{
QUEEN'S
UNIVERSITY
BELFAST
}

\section{Cattle movements in Northern Ireland form a robust network: implications for disease management}

Brown, E., Marshall, A. H., Mitchell, H. J., \& Bryne, A. W. (2019). Cattle movements in Northern Ireland form a robust network: implications for disease management. Preventive veterinary medicine, 170, [104740].

https://doi.org/10.1016/j.prevetmed.2019.104740

\section{Published in:}

Preventive veterinary medicine

\section{Document Version:}

Peer reviewed version

\section{Queen's University Belfast - Research Portal:}

Link to publication record in Queen's University Belfast Research Portal

\section{Publisher rights}

Copyright 2019 Elsevier Ltd

This manuscript is distributed under a Creative Commons Attribution-NonCommercial-NoDerivs License

(https://creativecommons.org/licenses/by-nc-nd/4.0/), which permits distribution and reproduction for non-commercial purposes, provided the author and source are cited.

\section{General rights}

Copyright for the publications made accessible via the Queen's University Belfast Research Portal is retained by the author(s) and / or other copyright owners and it is a condition of accessing these publications that users recognise and abide by the legal requirements associated with these rights.

Take down policy

The Research Portal is Queen's institutional repository that provides access to Queen's research output. Every effort has been made to ensure that content in the Research Portal does not infringe any person's rights, or applicable UK laws. If you discover content in the Research Portal that you believe breaches copyright or violates any law, please contact openaccess@qub.ac.uk. 


\section{Manuscript Details}

\section{Manuscript number}

Title
PREVET_2018_533_R3

Cattle movements in Northern Ireland form a robust network: implications for disease management.
Research Paper

\section{Article type}

\section{Abstract}

The movements of undetected infected animals can facilitate long-distance pathogen spread, making control and eradication difficult by (re)infecting disease-free populations. Characterising movement patterns is essential in understanding pathogen spread and how potential interventions, particularly animal movement restrictions, could help as a control mechanism. In Northern Ireland ( $\mathrm{NI})$, cattle movements are important contributors to a significant portion of agricultural trade. They can be disrupted due to statutory interventions, for example, during bovine tuberculosis (bTB) control. Identifying populations at risk of becoming infected would allow for improved resource allocation. This could be through targeting herds with an above-average risk of becoming infected or spreading (amplifying) infection, and restricting their movement to manage future outbreaks. In this study, cattle movements were investigated using social network analysis (SNA) at the monthly temporal scale across NI during 2010-2015. Targeted and random herd restrictions were compared and their impact on the structure and connectivity of the networks' was assessed (e.g. connected component subgraphs). This work was contextualised in relation to bTB, the most persistent infectious disease currently impacting agriculture in $\mathrm{NI}$, where reduced connectivity would represent potential reduced vulnerability from infection introduction. There was seasonal variation in network size and level of connectivity with spring and autumn being the largest and most connected due to common farming practices in NI. Across the study period, there was limited inter-annual variation in global network metrics. On average there were 6.28 movements between each pair of nodes each month, low reciprocity (mean of 0.155 ) and the networks were moderately accessible with an average path length of 4.28. Movements were not confined to within each disease management area but frequently occurred between these areas (mean assortativity of -0.0731) and herds with high degree interacted with herds of low degree (mean assortativity of -0.351 ). The Giant Weakly Connected Component (GWCC) spanned most of the networks (between $75 \%$ and $100 \%$ of nodes); however the Giant Strongly Connected Component (GSCC) included, at most, $23 \%$ of the network. There was heterogeneous contributions across NI with little participation in the GSCC from some disease management areas, and the GSCC was comprised predominantly of 'beef breeders', 'beef rearers', and 'other/mixed' type herds. Targeted restrictions were more effective at fragmenting the network than randomly restricting movements when $25 \%$ of nodes or more were removed. Cattle networks in $\mathrm{NI}$ are extremely interconnected and robust to movement restrictions, suggesting potential vulnerability to movementfacilitated pathogen

Keywords

\section{Corresponding Author \\ Corresponding Author's Institution}

Order of Authors

Suggested reviewers
Northern Ireland; social network analysis; trade networks; disease control modelling; movement structure; seasonal fragmentation

\section{Emma Brown}

Queen's University Belfast

Emma Brown, Adele Marshall, Hannah Mitchell, Andrew Byrne spread, such as bTB. 


\section{Submission Files Included in this PDF}

File Name [File Type]

PrevVetMedCoverLetter.docx [Cover Letter]

PVMThirdResponseLetter.docx [Response to Reviewers]

CattleMovementPaper.docx [Manuscript File]

Figure1RevisedChordDiagram.jpeg [Figure]

Figure2RevisedGSCC.jpg [Figure]

Figure3RevisedGWCC.jpg [Figure]

Figure4RevisedNoComps.jpeg [Figure]

RevisedFigureCaptions.docx [e-Component]

Supplementary Material.docx [Supplementary Material]

To view all the submission files, including those not included in the PDF, click on the manuscript title on your EVISE Homepage, then click 'Download zip file'.

\section{Research Data Related to this Submission}

There are no linked research data sets for this submission. The following reason is given:

The authors do not have permission to share data 

1 Cattle movements in Northern Ireland form a robust network: implications

2 for disease management

3 Emma Brown*a, b, Adele H. Marshall ${ }^{a}$, Hannah J. Mitchell ${ }^{a}$, Andrew W. Byrne ${ }^{b, c^{\wedge}}$

$4 \quad$ a Mathematical Sciences Research Centre, Queen's University Belfast

$5 \quad$ b Veterinary Sciences Division, Agri-Food and Biosciences Institute, Belfast

6 c School of Biological Sciences, Queen's University Belfast

$7 \quad$ *Corresponding author: Emma Brown, Mathematical Sciences Research Centre, Queen's University

8 Belfast, University Road, Belfast, BT7 1NN. Email: ebrown99@qub.ac.uk Phone: +44 (0) 289097

$9 \quad 1923$

10

^ Current address: One-Health Unit, Department of Agriculture, Food and the Marine, Agriculture 11 House, Kildare Street, Dublin 2, Ireland.

\section{Abstract}

13 The movements of undetected infected animals can facilitate long-distance pathogen spread,

14 making control and eradication difficult by (re)infecting disease-free populations. Characterising

15 movement patterns is essential in understanding pathogen spread and how potential interventions, particularly animal movement restrictions, could help as a control mechanism. In Northern Ireland

17 (NI), cattle movements are important contributors to a significant portion of agricultural trade. They can be disrupted due to statutory interventions, for example, during bovine tuberculosis (bTB) control. Identifying populations at risk of becoming infected would allow for improved resource 20 allocation. This could be through targeting herds with an above-average risk of becoming infected or 21 spreading (amplifying) infection, and restricting their movement to manage future outbreaks. 
temporal scale across NI during 2010-2015. Targeted and random herd restrictions were compared and their impact on the structure and connectivity of the networks' was assessed (e.g. connected component subgraphs). This work was contextualised in relation to bTB, the most persistent 26 infectious disease currently impacting agriculture in $\mathrm{NI}$, where reduced connectivity would represent 27 potential reduced vulnerability from infection introduction.

There was seasonal variation in network size and level of connectivity with spring and autumn being the largest and most connected due to common farming practices in NI. Across the study period, there was limited inter-annual variation in global network metrics. On average there were 6.28 movements between each pair of nodes each month, low reciprocity (mean of 0.155 ) and the networks were moderately accessible with an average path length of 4.28. Movements were not confined to within each disease management area but frequently occurred between these areas (mean assortativity of -0.0731) and herds with high degree interacted with herds of low degree (mean assortativity of -0.351). The Giant Weakly Connected Component (GWCC) spanned most of the networks (between $75 \%$ and $100 \%$ of nodes); however the Giant Strongly Connected Component

(GSCC) included, at most, $23 \%$ of the network. There was heterogeneous contributions across $\mathrm{NI}$ with little participation in the GSCC from some disease management areas, and the GSCC was comprised predominantly of 'beef breeders', 'beef rearers', and 'other/mixed' type herds. Targeted restrictions were more effective at fragmenting the network than randomly restricting movements when $25 \%$ of nodes or more were removed. Cattle networks in NI are extremely interconnected and 42 robust to movement restrictions, suggesting potential vulnerability to movement-facilitated 43 pathogen spread, such as bTB. Northern Ireland, social network analysis, trade networks, disease control modelling, movement structure, seasonal fragmentation 
Animal movements are commonly found to be important facilitators of infection spread between spatially distinct groups, particularly the long-distance transportation of exposed hosts (El Allaki, et al., 2016; Enright and O'Hare, 2016). In the United Kingdom, long-distance spread was particularly important in the Foot and Mouth Disease (FMD) outbreak of 2001 where the disease spread quickly via animal movements (Gilbert, et al., 2005; Ortiz-Pelaez, et al., 2006). The introduction of infection through the trade of undiagnosed infected animals can initiate or prolong outbreaks. Understanding these dynamics is central to epidemiological modelling, and Social Network Analysis (SNA) is one approach which has assisted in uncovering the impact of influential "nodes" across complex 56 networks (Albert, et al., 2000; Robinson and Christley, 2007; Mweu, et al., 2013; Palisson, et al., 2016).

Social network analysis has been increasingly used to represent the spread of infectious diseases by representing social interactions as a transmission route (see Dube, et al., 2011 for a detailed overview). Such networks represent farms as nodes that are connected to each other by cattle movements (batched with respect to time interval or singular) as edges. The overall behaviour of the herds can be derived from assessing the dynamics of such networks, as well as gaining insight on how vulnerable the networks are to herds with an above-average number of connections. Highly connected herds have been previously found to have the potential to spread disease more effectively than the averagely connected herd (Natale, et al., 2009; Grear, et al., 2014; Dutta, et al., 2014; Hidano, et al., 2016; Vidondo and Voekl, 2018). The identification of these influential herds, or so-called 'superspreaders' (VanderWaal, et al., 2016), allows for targeted restrictions to be implemented. Doing so could reduce the potential risk of disease spread as this outcome was previously found when 'superspreaders' were excluded from networks resulting in a decreased potential outbreak size (Robinson and Christley, 2007; Mweu, et al., 2013). Movement restriction of 
71 high-risk herds is an important emergency tool employed for highly contagious outbreaks such as

72 FMD, in order to corral disease spread (Gibbens, et al., 2001). Exploitation of the networks'

73 characteristics for the purpose of disease control may be necessary when combating endemic

74 diseases which need a range of measures acting simultaneously before the disease is effectively

75 managed (Brooks-Pollock, et al., 2014). Such control measures have been investigated through the

76 restriction of movements of cattle from high-risk areas (Natale, et al., 2009; Grear, et al., 2014;

77 Dutta, et al., 2014; Ribeiro-Lima, et al., 2015; Hidano, et al., 2016). Furthermore, some real-world 78 networks, for example pig networks in Denmark, have also been found to exhibit characteristics that 79 can facilitate disease management (Bigras-Poulin, et al., 2006; Mweu, et al., 2013).

80 Bovine Tuberculosis (bTB), a bacterial infection caused by Mycobacterium bovis, is of particular high81 priority in NI (Abernethy, et al., 2006). Due to its chronic and multifactorial nature, bTB is endemic in

$82 \mathrm{NI}$ and has multiple transmission routes (Denny and Wilesmith, 1999; Abernethy, et al., 2006;

83 Broughan, et al., 2016), including cattle movement, which have been shown to be an influential risk

84 factor (Allen, et al., 2018). Herd incidence levels have fluctuated over the last decade, however, there has been a recent increase with incidence currently reported to be $9.22 \%$ in 2018 by the competent authority (DAERA: Department of Agriculture, Environment, and Rural Affairs, 2019). The agriculture industry in NI has been affected by bTB through decreased animal production, decreased 88 trade, and compensation for detected cattle (Abernethy, et al., 2006). In NI, temporary movement

89 restrictions also form part of the mandatory bTB control programme, in line with EU guidelines since

1964, when infected cattle are detected (though ante-mortem testing using the Single Intradermal 91 Comparative Cervical Tuberculin (SICCT) test and/or post-mortem confirmation of infection) 92 (Abernethy, et al., 2006). 
control measures such as pre-movement testing are not part of the standard bTB prevention procedure (Abernethy, et al., 2006). Despite the current use of trade restrictions, there is still a risk for pathogens to be dispersed through animal trade due to the moderate sensitivity of statutory diagnostic tests (e.g. estimated test sensitivity for bTB tuberculin skin test in infected herds in NI is 98 50-60\%; Lahuerta-Marin, et al., 2018). Indeed, because of this, some authors suggest there may be 99 a hidden burden of infection in endemically infected populations (Conlan, et al., 2012).

Understanding cattle movements and the networks they form is key to improving our knowledge of the risk for endemic disease spread, including bTB, in NI. Furthermore, the characteristics of such 102 networks allow us to infer the potential vulnerability of the network to infection spread.

This research had three aims: characterising the structure of cattle movements, identifying influential nodes, and assessing the efficacy of hypothetical movement control measures to tackle between-herd transmission. Network measures that identify influential nodes were used to apply targeted movement restrictions to the networks where these results were then compared with 107 randomly applied movement restrictions. The reduction of network connectivity was used as the 108 measurement of comparison.

\section{Methods}

111 Several network metrics were calculated to characterise movements in this paper, which were:

112 average path length, diameter, density, reciprocity, mean number of movements between each 113 distinct pair of nodes, number of components, the size of the Giant Weakly Connected Component 114 (GWCC) and the size of the Giant Strongly Connected Component (GSCC). These network metrics follow the standard definitions as defined by Dube, et al., (2009) and Martínez-López, et al., (2009). 
The degree and betweenness centrality distributions were also calculated with node degree and 117 betweenness centrality having a pivotal role in representing targeted movement restrictions.

118 Degree identifies the highly connected herds and the degree distribution that describes the population's distribution of infection risk. The degree distribution was fitted to the power law and log-normal distributions to assess the networks' connectivity structure. If the degree distribution 121 follows the power-law distribution, infection risk from movements can be considered heterogeneous

122 (Ribeiro-Lima, et al., 2015). Networks with a power-law degree distribution are defined as scale-free which have a positively skewed degree distribution and a relatively small group of highly connected nodes (Diestel, 2006; Mweu, et al., 2013; Vidondo and Voekl, 2018). Previous research has found 125 that scale-free networks are robust to random node removal but vulnerable when important nodes, 126 measured with degree and betweenness centrality, are removed (Albert, et al., 2000).

Networks are comprised of connected components, where nodes are grouped together if they are more connected to each other than to nodes from other components. Giant components can arise if they encompass a greater number of nodes and can be defined as strongly or weakly connected. If a Giant Strongly Connected Component (GSCC) exists then all nodes that reside within it can reach every other node in the component following the direction of the edges. If each node can reach every other node in the giant component when directionality is neglected then a Giant Weakly Connected Component (GWCC) exists (Albert, et al., 2000; Diestel, 2006). As nodes in a giant component have higher connectedness than nodes outside of the component, limiting the size of these giant components is of particular interest. Reduction of the size of giant components reduces the connectivity of the network, thus reducing the vulnerability of nodes to disease introduction

137 through cattle movements. As movement directionality is accounted for in the GSCC, it is particularly 
be the lower bound of the size of an epidemic (Kao, et al., 2006). Identifying efficient methods in fragmenting the GSCC is of particular importance given the need to reduce disease risk while maintaining economic optimality. Observing the number of weakly connected components of a network can also be used in conjunction with the GSCC as the GWCC is also an important measure due to its use as the inferred upper bound of an epidemic size (Motta, et al., 2017). Using both of 144 these measures provides an estimation of the size of an epidemic if one was introduced to the

network.

\section{Application to data}

The anonymised data used in this study was provided by DAERA from their Animal and Plant Information System (APHIS) database which included animal ID, date of movement, ID of previous herd, ID of the current herd and the movement type between 2010 and 2015. The animal IDs were unique to each individual and each registered herd also had its own ID number. The movement type can be defined as one of 13 possible types, however the most widely used types were (in descending order): standard trade between farms and markets, abattoir, and lairage movements. As of 2015 there were 33,688 registered herds observed in the dataset. It should be noted, however, that not all 33,688 herds participated in cattle trade between 2010 and 2015 as they may not have been active during this time or were operating as closed herds (did not trade cattle but remained active). Hence, only the registered herds that participated in cattle trade were included in the analysis.

The movements were batched with respect to their month and year, creating 72 observed networks, to reduce computational complexity (which increases with increasing data resolution) and to investigate how movements changed throughout and between each year. As DAERA records each movement in relation to individual cattle, cattle whose movement records had the same originating and destination herds for each month and year were aggregated together. Therefore, edges between nodes existed if at least one movement, batched with respect to their timestamp (month 
and year) between them existed. The number of movements that were batched together formed the

164 weight of the edge. The weighted edges were assessed through the mean number of movements between each pair of nodes as an additional network characteristic along with those in Table 1. directional with animal movements, the analysis considered only directed networks in the study. 168 This was to assess $\mathrm{NI}^{\prime}$ s cattle movements whilst also preserving their structure as the directionality of the movements could affect how the networks would behave under movement restrictions. Networks for each month in the study period were compared to the corresponding network in the other years to look for temporal consistency (little to no variation in network structure between the years of study). Nodes in these networks included standard farming premises, markets, lairage, and 173 abattoir (holding) herds to produce a comprehensive view of the movement patterns across the 174 region. To study how connectivity may be affected by herd restrictions, new sub-networks were created from the observed networks by removing nodes under different criteria at various thresholds. The three removal types were: randomly chosen nodes, targeting nodes with high betweenness centrality, and targeting nodes with high degree values. The betweenness centrality and degree metrics were used as they are indications of node influence or importance to network connectivity (Albert, et al., 2000). Removing nodes with high betweenness centrality and degree values may offer more effective network fragmentation as important nodes are more likely to be removed from the network. Removing these nodes would have the anticipated effect that the network would split into several components that would be more manageable for disease control (or act a buffers to disease spread). The thresholds at which nodes were removed were at $1 \%, 5 \%, 10 \%, 20 \%, 25 \%$, and $50 \%$. 
This was to observe the level required to greatly reduce network connectivity for each of the methods. Extreme thresholds were considered in this study to produce a wider view of the potential impact of movement restrictions. For targeted restrictions, the nodes were ranked in descending order with respect to their betweenness centrality and degree values. Due to the manner in which the observed networks were fragmented, degree and betweenness centrality were not recalculated when nodes were removed, as a new network was created at each removal threshold. This method was used to more effectively compare the effectiveness of random and targeted removal at the predefined thresholds. Scale-free networks have previously been found to be robust against high levels of random node removal but have fragmented into smaller components when a relatively small proportion of nodes were targeted with respect to their influence upon the network (Albert, et al., 2000; VanderWaal, et al., 2016). If the networks in $\mathrm{NI}$ are found to be scale-free, it would be anticipated that targeting influential nodes will show an improvement over random movement 197 restrictions as in Albert, et al., (2000).

All observed data for the metrics, apart from the average path length and diameter, were found to be normally distributed. The difference between these normal metrics and the values produced by the restricted networks were tested using the t-test at the $5 \%$ significance level. As the average path length and diameter were not normal, the differences were tested using the Mann-Whitney test at the $5 \%$ significance level. The metrics calculated for the manipulated networks were compared to 203 the metrics produced by the corresponding observed data.

When conducting single comparisons of the observed and manipulated networks, $1728 \mathrm{t}$-tests (72 observed networks*8 metrics*3 removal methods) were performed, necessitating the use of the 206 Bonferroni correction to adjust for Type 1 errors. The new critical value for the tests is

$$
\begin{gathered}
\alpha \text { Crit } 0.05 \\
\alpha_{\text {NewCrit }}=N=172 \overline{8=2.89} * 10^{-5}
\end{gathered}
$$


To investigate the possibility of Type 2 errors, the power of the tests was calculated using the 'stats'

Type 2 errors were expected to occur.

211 All network metrics, manipulations, and power-law fits were completed in R (R Core Team, 2019)

212 using the 'igraph' package, fits to the log-normal distribution were conducted with the 'goft' package

213 (Gonzalez-Estrada and Villasenor-Alva, 2017), figures were created using the 'ggplot2' package, and

214 the remaining statistical tests were undertaken in base R in RStudio Version 1.0.153 (Csardi and

215 Nepusz, 2006; R Core Team, 2019; Wickham, 2009).

216 Results

\section{Movement statistics}

218 Table 1: Mean (standard deviation) network metrics for each year in the study period. Each year had a network built for each month.

220

\begin{tabular}{|l|l|l|l|l|l|l|}
\hline Year & Density & Diameter & $\begin{array}{l}\text { Average } \\
\text { path length }\end{array}$ & Reciprocity & $\begin{array}{l}\text { Mean } \\
\text { movement } \\
\text { frequency } \\
\text { between node } \\
\text { pairs }\end{array}$ & $\begin{array}{l}\text { Number of } \\
\text { components } \\
\text { in network }\end{array}$ \\
\hline 2010 & $\begin{array}{l}1.60 * 10^{-4} \\
\left(2.14 * 10^{-5}\right)\end{array}$ & $13(0.739)$ & $4.35(0.144)$ & $\begin{array}{l}0.148 \\
(0.0251)\end{array}$ & $6.27(0.559)$ & $250(65)$ \\
\hline 2011 & $\begin{array}{l}1.55^{*} 10^{-4} \\
\left(1.66 * 10^{-5}\right)\end{array}$ & $13(0.965)$ & $4.30(0.104)$ & $\begin{array}{l}0.150 \\
(0.0187)\end{array}$ & $6.24(0.542)$ & $225(47)$ \\
\hline 2012 & $\begin{array}{l}1.58 * 10^{-4} \\
\left(1.63 * 10^{-5}\right)\end{array}$ & $13(1.30)$ & $\begin{array}{l}4.28 \\
(0.0964)\end{array}$ & $\begin{array}{l}0.156 \\
(0.0217)\end{array}$ & $6.32(0.519)$ & $228(36)$ \\
\hline 2013 & $\begin{array}{l}1.62 * 10^{-4} \\
\left(1.78 * 10^{-5}\right)\end{array}$ & $12(0.937)$ & $\begin{array}{l}4.26 \\
(0.0922)\end{array}$ & $\begin{array}{l}0.165 \\
(0.0243)\end{array}$ & $6.54(0.463)$ & $208(47)$ \\
\hline 2014 & $\begin{array}{l}1.65 * 10^{-4} \\
\left(2.00 * 10^{-5}\right)\end{array}$ & $13(1.08)$ & $\begin{array}{l}4.26 \\
(0.0914)\end{array}$ & $\begin{array}{l}0.156 \\
(0.0198)\end{array}$ & $6.18(0.512)$ & $221(48)$ \\
\hline
\end{tabular}




\begin{tabular}{|l|l|l|l|l|l|l|}
\hline 2015 & $1.58 * 10^{-4}$ & $13(1.28)$ & $\begin{array}{l}4.22 \\
\left(1.67 * 10^{-5}\right)\end{array}$ & $\begin{array}{l}0.156 \\
(0.0206)\end{array}$ & $6.11(0.428)$ & $227(52)$ \\
\hline
\end{tabular}

221

222

223

As there were 72 networks in total over the study period, Table 1 shows the yearly average of the calculated metrics. The metrics were predominantly consistent over the study period with little between-year variation, implied to be seasonal variation as shown in in Figures S1-S8 of the Supplementary Material. The network metrics were tested using the Shapiro-Wilk test for normality with only average path length and diameter having statistically significant p-values $\left(3.76^{*} 10^{-4}\right.$ and 227 $1.27 * 10^{-5}$ respectively) and the rest of the metrics failing to reject the null hypothesis that they are 228 normally distributed.

On average, there were 6.28 movements between each directly connected pair of nodes every month and low mean reciprocity of 0.155 . The networks were moderately accessible with an average path length of 4.28 between nodes, and mean diameter of 12.8 despite an average number 232 of 10,135 nodes participating in the networks. As displayed by S1-S8 in the Supplementary Material,

there was within-year variation for all metrics with clear seasonal patterns. Summarising the information from S1-S8, table 1 shows how the average yearly network density and diameter varies little throughout the study period while the average yearly number of components decreases with each year. As the study period progresses the standard deviation for the diameter decreases, shown in Table 1, while the standard deviation for average path length and the mean number of between238 node movements decreases. Table 1 also displays how the number of distinct components decrease over the study period, however the seasonal variation remains consistent (as shown by S1-S8). Networks in 2013 are shown, in Table 1, to have a smaller diameter, increased reciprocity, and

241 increased frequencies of movements between node pairs than networks of other years. However,

242 Table 2 shows that 2013's networks do not have the maximum number of nodes and edges, 243 suggesting that the nodes are connected in a more efficient manner. 
The number of nodes participating in the network fluctuated over the study period along with the

245

246

247

248

249

250

251

252

253

254

$$
255
$$

S9-S14, were also either 'beef breeding', 'beef rearing', or 'other' (which are mixed type and

unknown types).

Table 2: Mean (standard deviation) number of nodes and edges along with the size of the GSCC and 258 GWCC and their respective proportions for each year in the study period. Number of edges relates to 259 the number of batched cattle movements per monthly network.

\begin{tabular}{|l|l|l|l|l|l|l|}
\hline Year & $\begin{array}{l}\text { Number of } \\
\text { nodes }\end{array}$ & $\begin{array}{l}\text { Number of } \\
\text { edges }\end{array}$ & $\begin{array}{l}\text { Size of the } \\
\text { GSCC }\end{array}$ & $\begin{array}{l}\text { Size of the } \\
\text { GWCC }\end{array}$ & $\begin{array}{l}\text { Proportion } \\
\text { of network in } \\
\text { GSCC (95\% } \\
\text { Cl) }\end{array}$ & $\begin{array}{l}\text { Proportion of } \\
\text { network in } \\
\text { GWCC }(95 \% \\
\text { Cl) }\end{array}$ \\
\hline 2010 & $9,818(1,821)$ & $\begin{array}{l}15,266 \\
(3,656)\end{array}$ & $1,597(549)$ & $\begin{array}{l}9,269 \\
(1,957)\end{array}$ & $\begin{array}{l}16.3 \%(10.7, \\
21.9) \%\end{array}$ & $\begin{array}{l}94.4 \%(74.5, \\
100) \%\end{array}$ \\
\hline 2011 & $\begin{array}{l}10,443 \\
(1,524)\end{array}$ & $\begin{array}{l}16,723 \\
(3,093)\end{array}$ & $1,813(474)$ & $\begin{array}{l}9,952 \\
(1,609)\end{array}$ & $\begin{array}{l}17.4 \%(12.8, \\
21.9) \%\end{array}$ & $\begin{array}{l}95.3 \%(79.9, \\
100) \%\end{array}$ \\
\hline 2012 & $\begin{array}{l}10,100 \\
(1,345)\end{array}$ & $\begin{array}{l}15,976 \\
(2,640)\end{array}$ & $1,713(379)$ & $\begin{array}{l}9,603 \\
(1,407)\end{array}$ & $\begin{array}{l}17.0 \% \\
(13.2,20.7) \%\end{array}$ & $\begin{array}{l}95.1 \%(81.1, \\
100) \%\end{array}$ \\
\hline 2013 & $\begin{array}{l}10,102 \\
(1,576)\end{array}$ & $\begin{array}{l}16,379 \\
(3,327)\end{array}$ & $1,771(527)$ & $\begin{array}{l}9,652 \\
(1,668)\end{array}$ & $\begin{array}{l}17.5 \%(12.3, \\
22.7) \%\end{array}$ & $\begin{array}{l}95.5 \%(79.0, \\
100) \%\end{array}$ \\
\hline 2014 & $\begin{array}{l}10,010 \\
(1,698)\end{array}$ & $\begin{array}{l}16,291 \\
(3,504)\end{array}$ & $1,669(521)$ & $\begin{array}{l}9,529 \\
(1,785)\end{array}$ & $\begin{array}{l}16.7 \%(11.5, \\
21.9) \%\end{array}$ & $\begin{array}{l}95.2 \%(77.4, \\
100) \%\end{array}$ \\
\hline 2015 & $\begin{array}{l}10,334 \\
(1,433)\end{array}$ & $\begin{array}{l}16,773 \\
(3,065)\end{array}$ & $1,735(524)$ & $\begin{array}{l}9,842 \\
(1,510)\end{array}$ & $\begin{array}{l}16.8 \%(11.7, \\
21.9) \%\end{array}$ & $\begin{array}{l}95.2 \%(80.6, \\
100) \%\end{array}$ \\
\hline
\end{tabular}


261 Cattle movements were not confined to any one geographic area of $\mathrm{NI}$, however there was heterogeneity in the proportion of movements occurring across the DVOs (10 District Veterinary Offices for administrative purposes; Fig. S15 of the Supplementary Material) of NI. Figure 1 and Table 3 show that Dungannon DVO had the highest proportion of movements originating and entering into it, while Derry DVO had the smallest proportion of movements originating and entering

it. The assortativity of the networks was calculated with respect to the DVO of participating nodes and also their degree. Negative values infer that movements do not tend to occur within the same group (DVO or degree) while positive results show otherwise. The mean assortativity value with respect to DVO was -0.0731 suggesting that there was a moderate level of mixing between DVOs that is also observed in Figure 1. Figure 1 highlights how there is a high level of between-DVO movements throughout NI with over half of the DVOs interacting with all other DVOs in NI. There was also moderate mixing between nodes of different degree values with a mean value of -0.351 , 273 implying that highly connected nodes did not only interact with other highly connected nodes, so 274 low-degree nodes are not necessarily at low-risk.

Table 3: Proportion of movements in NI that start and end in each DVO.

\begin{tabular}{|l|l|l|}
\hline DVO & $\begin{array}{l}\text { Proportion of movements that } \\
\text { originate in the DVO }\end{array}$ & $\begin{array}{l}\text { Proportion of movements that } \\
\text { end in the DVO }\end{array}$ \\
\hline Armagh & 0.113 & 0.156 \\
\hline Ballymena & 0.113 & 0.130 \\
\hline Coleraine & 0.104 & 0.0932 \\
\hline Derry & 0.0163 & 0.0178 \\
\hline Dungannon & 0.157 & 0.218 \\
\hline Enniskillen & 0.143 & 0.107 \\
\hline Mallusk & 0.0741 & 0.0448 \\
\hline Newry & 0.0830 & 0.0769 \\
\hline Newtownards & 0.0553 & 0.0363 \\
\hline Omagh & 0.141 & 0.121 \\
\hline
\end{tabular}

277 Table 4: Mean (Standard Deviation) Assortativity Index of the monthly networks during 2010-2015.

\begin{tabular}{|l|l|l|}
\hline Year & Assortativity of DVO & Assortativity of Degree \\
\hline
\end{tabular}




\begin{tabular}{|l|l|l|}
\hline 2010 & -0.0842 & -0.334 \\
\hline 2011 & -0.0972 & -0.333 \\
\hline 2012 & -0.112 & -0.344 \\
\hline 2013 & -0.122 & -0.357 \\
\hline 2014 & -0.0978 & -0.368 \\
\hline 2015 & 0.0740 & -0.374 \\
\hline
\end{tabular}

278

The networks' degree distributions were tested (plotted in figures S16-S21 of the Supplementary

Material), at the $5 \%$ level of significance, for their fit to the power-law distribution and the $\log 281$

normal distribution with each distribution being the hypothesised distribution of the data. All p282

values for the power-law distribution were non-significant (all p-values>0.9; Table 5); $p$-values for

the log-normal distribution were extremely significant (all $p$-values $<0.0001 ;$ Table 5 ). Thus, the

hypothesis that the degree distribution was power-law distributed failed to be rejected while the

test for the log-normal distribution was rejected. Thus, the networks were considered to be

scale286 free and were subsequently expected to be more vulnerable to targeted node

removal than random

node removal.

Table 5: Mean (standard deviation) p-values testing the null hypothesis that the data follows (a) the 289 power law distribution and (b) the log-normal distribution.

\begin{tabular}{|l|l|l|}
\hline Year & Power-law distribution $p$-value & $\begin{array}{l}\text { Log-normal distribution } p \text { - } \\
\text { value }\end{array}$ \\
\hline 2010 & $0.941(0.200)$ & $8.08 * 10^{-26}\left(2.80 * 10^{-25}\right)$ \\
\hline 2011 & $0.995(0.0132)$ & $5.65 * 10^{-26}\left(1.41 * 10^{-29}\right)$ \\
\hline 2012 & $0.999(0.000974)$ & $5.60 * 10^{-31}\left(1.92 * 10^{-30}\right)$ \\
\hline 2013 & $1.00(0.000274)$ & $1.08 * 10^{-26}\left(3.73 * 10^{-26}\right)$ \\
\hline 2014 & $0.999(0.000672)$ & $6.54 * 10^{-25}\left(2.26 * 10^{-24}\right)$ \\
\hline 2015 & $0.999(0.000747)$ & $2.04 * 10^{-25}\left(7.06 * 10^{-25}\right)$ \\
\hline
\end{tabular}


Influential nodes were identified using the degree and normalised betweenness centrality

distributions. Influential nodes were chosen as those with a total degree of 100 or greater, or nodes

with a normalised betweenness centrality of 0.01 or greater. These thresholds were chosen through visual inspection of the distributions (see figures S22-S27 of the Supplementary Material), where the majority of the nodes had a degree less than 100 and a normalised betweenness centrality of less than 0.01. Nodes with high degree resided in Armagh, Ballymena, Coleraine, Dungannon, Enniskillen,

Newtownards, and Omagh and were either markets, export, or mixed herds. Nodes with high

normalised betweenness centrality scores were from Armagh, Ballymena, Coleraine, Dungannon, 298 Enniskillen, and Omagh. There was a greater composition of herd types with markets, beef breeders, 299 beef rearers, beef fatteners, export, and mixed herds being identified.

\section{Comparison of node removal}

301 After identifying the network characteristics, movement restrictions were simulated by removing nodes from each of the 72 observed networks at 6 different thresholds under 3 different criteria. Thus, 1,296 new, manipulated networks were created and the network metrics were re-calculated

304 for each new manipulated network. The GSCC, GWCC, and number of components were used as measures of connectivity for the manipulated networks as their changes were the most interpretable and they are the easiest to use for disease control purposes. Figures 2-4 show the 307 changes in the size of the GSCC, size of the GWCC, and the number of components in the network 308 while S28-S32 display the rest of the metrics in the Supplementary Material.

309 Table 6: Mean (standard deviation) reduction in GSCC and GWCC size under the 3 criteria at the 25\% 310 removal threshold.

\begin{tabular}{|l|l|l|l|l|l|l|}
\hline Year & $\begin{array}{l}\text { GSCC- } \\
\text { Betweenness }\end{array}$ & GSCC- Degree & $\begin{array}{l}\text { GSCC- } \\
\text { Random }\end{array}$ & $\begin{array}{l}\text { GWCC- } \\
\text { Betweenness }\end{array}$ & $\begin{array}{l}\text { GWCC- } \\
\text { Degree }\end{array}$ & $\begin{array}{l}\text { GWCC- } \\
\text { Random }\end{array}$ \\
\hline 2010 & $53 \%(16 \%)$ & $50 \%(17 \%)$ & $41 \%(24 \%)$ & $43 \%(12 \%)$ & $42 \%(14 \%)$ & $35 \%(15 \%)$ \\
\hline 2011 & $49 \%(20 \%)$ & $52 \%(19 \%)$ & $39 \%(17 \%)$ & $40 \%(15 \%)$ & $42 \%(14 \%)$ & $33 \%(12 \%)$ \\
\hline 2012 & $49 \%(16 \%)$ & $45 \%(16 \%)$ & $44 \%(16 \%)$ & $40 \%(12 \%)$ & $38 \%(12 \%)$ & $37 \%(11 \%)$ \\
\hline
\end{tabular}




\begin{tabular}{|l|l|l|l|l|l|l|}
\hline 2013 & $44 \%(19 \%)$ & $44 \%(19 \%)$ & $44 \%(19 \%)$ & $38 \%(12 \%)$ & $40 \%(13 \%)$ & $36 \%(12 \%)$ \\
\hline 2014 & $43 \%(20 \%)$ & $46 \%(17 \%)$ & $42 \%(18 \%)$ & $36 \%(14 \%)$ & $38 \%(12 \%)$ & $35 \%(14 \%)$ \\
\hline 2015 & $49 \%(19 \%)$ & $47 \%(16 \%)$ & $43 \%(14 \%)$ & $40 \%(11 \%)$ & $39 \%(9 \%)$ & $35 \%(8 \%)$ \\
\hline
\end{tabular}

312 Table 7: Mean (standard deviation) reduction in the number of components under the 3 criteria at 313 the $25 \%$ threshold.

\begin{tabular}{|l|l|l|l|}
\hline Year & $\begin{array}{l}\text { Number of } \\
\text { components - } \\
\text { Betweenness }\end{array}$ & $\begin{array}{l}\text { Number of } \\
\text { components - Degree }\end{array}$ & $\begin{array}{l}\text { Number of } \\
\text { components- Random }\end{array}$ \\
\hline 2010 & $604 \%(204 \%)$ & $569 \%(82 \%)$ & $430 \%(98 \%)$ \\
\hline 2011 & $602 \%(220 \%)$ & $682 \%(154 \%)$ & $448 \%(80 \%)$ \\
\hline 2012 & $572 \%(220 \%)$ & $611 \%(96 \%)$ & $562 \%(122 \%)$ \\
\hline 2013 & $565 \%(70 \%)$ & $613 \%(110 \%)$ & $611 \%(165 \%)$ \\
\hline 2014 & $434 \%(74 \%)$ & $501 \%(44 \%)$ & $508 \%(57 \%)$ \\
\hline 2015 & $711 \%(134 \%)$ & $568 \%(86 \%)$ & $507 \%(98 \%)$ \\
\hline
\end{tabular}

314

The size of both the GSCC and GWCC, in Figures 2 and 3, decreased under all movement restrictions

316 and the removal criteria produced overlapping values until $25 \%$ of nodes were removed. Despite the

317 networks exhibiting scale-free properties which suggest that only a small number of targeted

318 restrictions would be necessary to effectively shrink components, this behaviour was not observed.

319 Targeted restrictions at the 5\% threshold only reduced the GSCC by between $7-20 \%$ in size and

320 reduced the GWCC in size by between $7-13 \%$ while random restrictions reduced the GSCC by

321 between $7-11 \%$ and the GWCC by $7-9 \%$. At the $25 \%$ threshold the targeted restrictions were more

322 effective in reducing the GSCC and GWCC, seen in Table 6, except for 2013 where the mean

323 reduction by random removal was the same as the targeted restrictions. Like the changed GSCC and

324 GWCC, the number of components (Figure 4) increased more effectively by targeted restrictions at 325 the $25 \%$ level apart from 2013 where random restrictions, on average, fragmented the network 326 more effectively.

\section{Comparison of the manipulated networks and the observed data}

328 The metric values produced by the manipulated networks were batched according to their 
corresponding observed network. As the Bonferroni correction reduced the significance threshold to

$2.86 * 10^{-5}$, there were only two significant $p$-values found. These were for differences in the

reciprocity of the December 2012 network and for the number of components in the January 2012 network when nodes were removed using degree. If fewer networks were assessed potentially more significant differences may become apparent allowing for comparisons between restriction criteria. 334 Therefore, the extensiveness of the study was one of its own limits.

The conventional acceptable power threshold is 0.8 (Ellis, 2010), and as expected from the number of conducted tests, the power values produced by the manipulated networks were mostly smaller than 0.8 . Out of 72 values only 4 were greater than 0.8 for the GSCC and for the GWCC, there was only 1 . For the number of components, 14 out of 72 values were an acceptable power value. There was only one acceptable power value each for reciprocity and diameter and the rest of the metrics had power values of less than 0.8 .

\section{Discussion}

342 Large-scale recording of animal movements has yielded considerable data sources to capture the

343 dynamics of domestic animal populations and has allowed for the development of modelling tools to

344 inform on important epidemiological processes like disease dissemination risk (VanderWaal, et al., 345 2017). From these data sources, important insights into the connectivity and robustness of these

networks to perturbations can be assessed. Within this research, a data rich resource on animal

347 movements was utilised to illustrate the characteristics of animal movement through trade across

348 NI. The networks were shown to be large, complex, and highly connected with little temporal 349 variation at the yearly temporal scale. This demonstrates the value in collecting data at both high 350 spatial and temporal resolution to aid in the control of infectious pathogens.

351 Overall the networks described in this study became larger and increasingly connected over time.

352 This increased connectivity raises the vulnerability of averagely connected nodes, as the chance of 
being indirectly connected to an influential node increases. The networks' structure however appears to be temporally consistent between years of study, reflecting the lack of significant global disruption (e.g. emerging disease outbreaks) in cattle movements during the study period. The observed networks exhibited some temporal consistency in the movement frequencies and metrics indicating that if disease measures were introduced then the most recent movement data may not be necessary (Vernon and Keeling, 2009). Given that many endemic diseases, like bTB, are already 359 difficult to control, it may be appropriate to use updated, near 'real-time', movement data to further 360 assist disease management, especially when such data are readily available.

Nodes in the networks were easily accessible due to the small values for the average path length and diameter (Table 1). Thus, cattle movements could be effective facilitators of infection in NI, even

to relatively unconnected nodes due to the moderate disassortivity of the network. There was some seasonal variation found in the networks' metrics, S1-S8 of the Supplementary Material, most likely due to farming practices (McClenaghan, 2010). Networks for the summer and winter were generally smaller and less connected likely due to summer grazing and winter housing practices, respectively (McClenaghan, 2010). Networks for April, May, and October, however, were denser, exhibited higher connectivity and had more batched movements occurring between their nodes (S1-S8). Thus,

herds would be more vulnerable to potential disease spread (via trade) in spring and autumn, especially for highly transmissible pathogens. This seasonal variation was evident across all years despite the decreasing number of distinct components over time (Table 1). Thus, while the potential

for disease to be introduced into herds is relatively consistent throughout the year (S1 -S8), the overall risk of disease introduction has increased (Table 1). Combining this with the result that more herds are participating and trading with an increased number of distinct partners, there is an $\mathbf{3 7 5}$ amplified risk of disease spreading through NI via cattle trade (Table 1). 
DAERAs official statistics show that the Newry DVO was the area that had the highest number of

distinct bTB herd breakdowns across the study period (DAERA, 2018). Dungannon DVO, however, was the area that was found to have the highest proportion of movements, not the Newry DVO, highlighting that overall network connectivity should also be considered, not just the proportion of movements. The heterogeneity of cattle trade participation across NI reinforces the need for targeted movement restrictions in the event of disease control implementation rather than a blanket restriction which would affect low-risk areas of NI. Targeting herd restrictions for particular DVOs may still disrupt the overall cattle trade as the assortativity showed that there was a moderate level of between-DVO mixing that can also be observed in Figure 1. Also given that most of the DVOs 385 in NI contained herds that were influential and/or were highly connected, disruption may indeed be 386 widespread. However since the assortativity was weak, restricting high-risk DVOs from moving cattle 387 to low-risk DVOs may still be a valid option in extreme cases.

Despite the majority of (at times all) nodes in the networks were weakly connected, the GSCC contained at most $23 \%$ of the network. Most of these nodes were 'beef breeders', 'beef rearers' and 'other/mixed', highlighting the variance in the connectivity of different herd types. These herd types, along with 'markets', 'beef breeders', 'beef fatteners', and 'export' types, were also found to be influential due to their high normalised betweenness centrality values, while 'markets' and 'mixed' herds also had high degree scores. Since these types of nodes are highly connected and are the most influential in terms of betweenness centrality and degree, fragmenting this component to 395 optimally target movement restrictions may be considered key. The heterogeneity in DVO membership of the GSCC over the study period also highlights that network connectivity is influential. Indeed, as suggested by other research, the GSCC represents an approximate lower bound of the maximal epidemic size (Kao, et al., 2006). In contrast, the GWCC represents the 399 potential upper bound (Motta, et al., 2017; up to $100 \%$ of nodes in this network).

All 72 networks were found to be best described by the power-law distribution, implying that the 
401

402

403

404

405

406

407

408

409

410

411

414

415

416

networks were all scale-free and that the networks would be robust against random node restrictions (Albert, et al., 2000). This was hypothesised to occur due to other animal networks possessing this characteristic when a relatively small group of nodes were found to be the most influential in the network (Mweu, et al., 2013; Grear, et al., 2014; Ribeiro-Lima, et al., 2015; VanderWaal, et al., 2016). Such networks are consistent with the concept of super-spreader herds/nodes existing across the network (VanderWaal, et al., 2016). Hence, it can also be stated that there are indeed a relatively small group of highly connected nodes, mostly residents of the networks' GSCC. High levels of connectivity disparity observed between individual nodes emphasises that homogeneous treatment of herds throughout the region may not be effective and could potentially affect herds with the least risk of infection (Dube, et al., 2011; Ribeiro-Lima, et al., 2015; VanderWaal, et al., 2016). The degree assortativity highlighted that highly connected nodes did not 412 only interact with other highly connected nodes, but with nodes that were relatively unconnected.

Hence, their potential to spread disease to averagely connected herds should be noted and lends weight to the need for targeted restrictions given that these highly connected herds would have a substantial role in disease introduction. The degree assortativity values also show the validity of assessing the effectiveness of targeted restrictions based on a herd's degree given the potential 417 implications of disease spread.

Markets were also, unsurprisingly, the most influential nodes in the networks of this study (also see Robinson and Christley, 2007) and future disease management should consider their potential importance in targeting interventions and spreading infection. The influence of markets can be particularly noticed in their high degree values and the increasing reciprocity as more cattle are moved between them towards the end of the production cycle - though like findings elsewhere, not all markets were equally as central or connected (Robinson and Christley, 2007). Markets have additionally been noted to be important in other movement networks with previous studies finding 
that the estimated outbreak size decreased when markets were removed from the network

importance of markets in terms of infection control should be considered for future research, especially when a highly connected network such as the one observed in NI exists. Beef-type herds were also found to be influential in this study and with a high cattle turnover rate they would have a higher vulnerability to infection introduction than other herd types. Dutta, et al., (2014) also demonstrated differences in the characteristics of dairy and beef sub-networks in France. Therefore, beef herds (beef breeders and finishers) should be considered differentially in disease management as they provided most of the cattle involved in the movements between the highly influential market nodes. Cattle moved from beef finishers may be considered lower risk when animals move straight to abattoir. However, not all animals move straight to abattoir but instead to lairage/holding herds 436 which can sometimes have further outward movements (potentially as a consequence of market 437 value changes).

Targeted restrictions of influential herds were demonstrated to become more effective in network fragmentation when $25 \%$ of the network was removed, which could interrupt chains of infection

440 (Natale, et al., 2009; Rautureau, et al., 2011; Buttner, et al., 2013). The improved effectiveness of targeted herd restrictions over random restrictions after this threshold highlighted the need to

442 account for the influence of market, beef breeder, and beef finisher herds when managing infectious

disease. This difference in implementation was attributed to the increased probability that an influential node connecting components would be removed under the criteria for the targeted methods. It was anticipated that several components would be created through the use of targeted 446 node removal in the network. While this did occur, the GSCC was still between $47-61 \%$ of its original size and the GWCC was largely unaffected staying around $85-92 \%$ of its original size at the $25 \%$ removal threshold, implying that despite the increased effectiveness of targeted restrictions, the 
cattle networks of $\mathrm{NI}$ are extremely interconnected and would not be easily fragmented for disease control purposes (in contrast with cattle movement networks elsewhere (Natale, et al., 2009; VanderWaal, et al., 2016)). Given the trading networks for cattle and other species in Europe (Natale, et al., 2009; Rautureau, et al., 2011; Buttner, et al., 2013), this level of connectedness in NI 453 may suggest that the system is particularly vulnerable if a highly transmissible pathogen was introduced.

Some European studies found that their animal networks contained GSCCs which disappeared when a relatively small proportion of nodes with the highest betweenness centrality values were targeted for removal (Rautureau, et al., 2011; Dutta, et al., 2014). Similarly, just 1\% of nodes with the highest degree values were needed to be targeted for removal for an $88 \%$ reduction in the number of infected nodes in a cattle network from Italy (Natale, et al., 2009). It appears the NI network exhibits an increased level of connectedness due to the relatively small GSCC (10.7-22.7\% of the network) that is still sizeable after movement restrictions. Direct comparisons across jurisdictions, however, are difficult due to the differing approaches to collecting movement data and geographical differences. This network structure may also be due to Nl's uniqueness in that it is a region on the periphery of Europe, has different protocols from the Republic of Ireland (ROI), and is physically separate from Great Britain (GB). Thus, the potential physical and legal constraints may have 466 resulted in a reliance on trade within the region (which is only $14,130 \mathrm{~km}^{2}$ in area) creating a highly 467 interconnected network of herds.

Our results imply that in NI targeted interventions lack the effectiveness that had been hypothesised and so movement restrictions would only be able to support other controls, instead of being a primary measure for disease control. For hypothesized emerging or high-risk disease incursions, a

471 range of controls or more draconian approaches may be required to contain spread.

472 There were some limitations with this work. For example, these networks were only built upon 
movements between premises and do not include movements within the premises. These withinherd movements can be frequent, and are important as many enterprises in NI exhibit farm fragmentation (conacre) as they are made up of two or more geographically distinct land parcels (E. Campbell, 2018, pers. comm.). Furthermore, for infectious diseases like bTB that also have a wildlife reservoir, i.e. the badger Meles meles (Allen, et al., 2018), spill-over could also occur from herds to 478 wildlife, which could impact on infection introduction in the local landscape (Byrne, et al., 2015).

The extensiveness of the study was also a limitation due to the increased likelihood of Type 1 (significance level was at $2.89 * 10^{* 5}$ ) and Type 2 errors occurring when comparing the manipulated networks with the corresponding observed data. Due to the lack of significant $p$-values in the analysis it is difficult to say which targeted method would have been more appropriate. However, given that degree is a straightforward, interpretable measure to be applied in control strategies, targeting highly connected herds may be more effective. In contrast, Rautureau, et al., (2011) found that betweenness centrality was the most efficient way to break-up components within the French cattle trade network. Future analyses could target time periods of interest to maximise their utility 487 and to be able to compare varying targeted interventions more effectively.

\section{Conclusion}

The overall network of cattle movements between herds in NI is extremely connected through animal movements and trade. This network has been shown to be robust to perturbations through the restriction of animal movement from selected herds. Even when a practical amount (25\%) of herds were targeted to maximise network fragmentation (targeted restriction), there was not the anticipated level of compartmentalisation across the network to potentially corral the spread of infectious pathogens. Some seasonal fragmentation, however, was observed to occur naturally due to the common regional farming practices. While seasonal fragmentation is only a consequence of 
these practices, it provides insight into herd connectivity and the identification of this potential exploit may form the basis of future disease management analyses. Incorporating the knowledge 498 gained from the network analysis would allow for a better understanding of herd vulnerability, and 499 also for herd importance across the network.

If movement controls are to be considered for implementation, using the herd's degree may be the most appropriate metric to use as degree is simpler to calculate and interpret than betweenness centrality. If a measure of connectivity was required to assess the network prior to disease management, the number of components, GSCC, and GWCC size would be useful metrics to assess connectivity and vulnerability. This is due to the use of component numbers as a measure of network fragmentation in this study. For the GSCC and GWCC, their use would be beneficial given their interpretation as approximate lower and upper bounds for an estimated epidemic size if such a disease was to be introduced (Kao, et al., 2006; Motta, et al., 2017). Targeted restrictions would 508 have a smaller than hypothesised effect on herd vulnerability but would potentially be useful if used 509 with other control measures.

510 Overall, the herds in NI are extremely interconnected to the extent that they would still be quite

511 vulnerable even if extensive targeted movement restrictions were implemented. While the assessment of NI's cattle movements showed that they could be used for disease management, to 513 further understand them, the risk of infection of diseases of critical importance (like bTB) would $514 \quad$ need to be analysed.

\section{Acknowledgement}

516 E. Brown is funded through a DAERA Postgraduate Studentship. The funders were not involved in

517 the study. The authors are grateful to DAERA for providing the data and to Jordan Graham for his 518 assistance with the data. Data used in this study was attained through a Data Share 
Agreement 519 between the authors and DAERA. The authors are also appreciative to the reviewers for their useful 520 comments which have improved the manuscript.

\section{References}

522 Abernethy, D.A., Denny, G.O., Menzies, F.D., McGuckian, P., Honhold, N., Roberts, A.R., 2006. The $523 \quad$ Northern Ireland programme for the control and eradication of mycobacterium bovis.

Vet. $524 \quad$ Microbiol. 112, 231-237.

525 Albert, R., Jeong, H., Barabasi, A., 2000. Error and attack tolerance of complex networks. Nature 406, 526 378-382.

527 Allen, A., Skuce, R., Byrne, A., 2018. Bovine tuberculosis in Britain and Ireland - A perfect storm? The 528 confluence of potential ecological and epidemiological impediments to controlling a chronic 529 infectious disease. Frontiers in Veterinary Science.

530 Bigras-Poulin, M., Thompson, R.A., Chriel, M., Mortensen, S., Greiner, M., 2006. Network analysis of 531 Danish cattle industry trade patterns as an evaluation of risk potential for disease spread. Prev. Vet. 532 Med. 76, 11-39.

533 Brooks-Pollock, E., Roberts, G.O., Keeling, M.J., 2014. A dynamic model of bovine tuberculosis spread 534 and control in Great Britain. Nature 511, 228-231.

535 Broughan, J.M., Judge, J., Ely, E., Delahay, R.J., Wilson, G., Clifton-Hadley, R.S., Goodchild, A.V., 536 Bishop, H., Parry, J.E., Downs, S.H., 2016. A review of risk factors for bovine tuberculosis infection in 537 cattle in the UK and Ireland. Epidemiol. Infect. 1-28.

538 Buttner, K., Krieter, J., Traulsen, A., Traulsen, I., 2013. Efficient interruption of infection chains by 539 targeted removal of central holdings in an animal trade network. PLoS ONE 8(9), e74292.

540 Byrne, A.W., Kenny, K., Fogarty, U., O’Keeffe, J.J., More, S.J., McGrath, G., Teeling, M., Martin, S.W., 541 Dohoo, I.R., 2015. Spatial and temporal analyses of metrics of tuberculosis infection in badgers 542 (Meles meles) from the republic of Ireland: Trends in apparent prevalence. Prev. Vet. Med. 122(3), $543 \quad 345-354$.

544 Conlan, A.J., McKinley, T.J., Karolemeas, K., Pollock, E.B., Goodchild, A.V., Mitchell, A.P., 545 Birch, C.P., Clifton-Hadley, R.S. and Wood, J.L., 2012. Estimating the hidden burden of 546 bovine tuberculosis in Great Britain. PLoS Computational Biology, 8(10), p.e1002730.

547 Csardi, G., Nepusz, T., 2006. The igraph software package for complex network research. 548 InterJournal Complex Systems, 1695.

549 DAERA, 2019. Department of agriculture, environment and rural affairs: Bovine tuberculosis monthly 550 statistics, Accessed: April 2019. 
551 Denny, G.O., Wilesmith, J.W., 1999. Bovine tuberculosis in Northern Ireland: A case-control study of

552 herd risk factors. The Veterinary Record.

553 Diestel, R., 2006. Graph Theory. Springer.

554 Dube, C., Ribble, C., Kelton, D., McNab, B., 2009. A review of network analysis terminology and its 555 application to foot-and-mouth disease modelling and policy development. Transbound. Emerg. Dis. 556 56(3), 73-85.

557 Dube, C., Ribble, C.F., Kelton, D.F., McNab, B., 2011. Introduction to network analysis and its 558 implications for animal disease modelling. Scientific and Technical Review (International Office of 559 Epizootics).

560 Dutta, B.L., Ezanno, P., Vergu, E., 2014. Characteristics of the spatio-temporal network of cattle 561 movements in France over a 5-year period. Prev. Vet. Med. 117, 79-94.

562 El Allaki, F., Harrington, N., Howden, K., 2016. Assessing the sensitivity of bovine tuberculosis 563 surveillance in Canada's cattle population, 2009-2013. Prev. Vet. Med. 134, 145-152.

564 Ellis, P.D., 2010. The Essential Guide to Effect Sizes: An Introduction to Statistical Power, Meta565 Analysis and the Interpretation of Research Results. Cambridge University Press.

566 Enright, J.A., O'Hare, A., 2016. Reconstructing disease transmission dynamics from animal

567 movements and test data. Stochastic Environmental Research and Risk Assessment 1-9.

568 Gibbens, J.C., Sharpe, C.E., Wilesmith, J.W., Mansley, L.M., Michalopoulou, E., Ryan, J.B., Hudson, M., 5692001. Descriptive epidemiology of the 2001 foot-and-mouth disease epidemic in Great Britain: The 570 first five months. Vet Rec 149 (24), 729-743.

571 Gilbert, M., Mitchell, A., Bourn, D., Mawdsley, J., Clifton-Hadley, R., Wint, W., 2005. Cattle 572 movements and bovine tuberculosis in Great Britain. Nature 435, 491-496.

573 Gonzalez-Estrada E., Villasenor-Alva, J.A., 2017. Goft: Tests of fit for some probability distributions. 574 CRAN repository.

575 Gorsich, E.E., Luis, A.D., Buhnerkempe, M.G., Grear, D.A., Portacci, K., Miller, R.S., Webb, C.T., 2016. 576 Mapping U.S. cattle shipment networks: Spatial and temporal patterns of trade communities from 5772009 to 2011. Prev. Vet. Med. 134, 82-91.

578 Grear, D.A., Kaneene, J.B., Averill, J.J., Webb, C.T., 2014. Local cattle movements in response to 579 ongoing bovine tuberculosis zonation and regulations in Michigan, USA. Prev. Vet. Med. 114, 201580212.

581 Hidano, A., Carpenter, T.E., Stevenson, M.A., Gates, M.C., 2016. Evaluating the efficacy of 582 regionalisation in limiting high-risk livestock trade movements. Prev. Vet. Med. 133, 31-41.

583 Kao, R., Danon, L., Green, D. \& Kiss, I., 2006. Demographic structure and pathogen dynamics on the 584 network of livestock movements in Great Britain. Proceedings of the Royal Society B: Biological 585 Sciences 273, 1999-2007. 
586 Lahuerta-Marin, A., Milne, M.G., McNair, J., Skuce, R.A., McBride, S.H., Menzies, F.D., 587 McDowell, S.J.W., Byrne, A.W., Handel, I.G. and Bronsvoort, B.D.C., 2018. Bayesian latent 588 class estimation of sensitivity and specificity parameters of diagnostic tests for bovine 589 tuberculosis in chronically infected herds in Northern Ireland. The Veterinary Journal, 238, 590 pp.15-21.

591 Martínez-López, B., Perez, A.M., Sánchez-Vizcaíno, J.M., 2009. Social Network Analysis.

592 Review of general concepts and use in preventive veterinary medicine. Transbound. Emerg. 593 Dis. 56(4), 109-20.

594 McClenaghan, R., 2010. Grazing management booklet, DAERA.

595 McHenry, P., 2018. Beef management booklet, DAERA.

596 Motta, P., Porphyre, T., Handel, I., Hamman, S.M., Ngwa, V.N., Tanya, V., Morgan, K., Christley, R. 597 and Barend, M., 2017. Implications of the cattle trade network in Cameroon for regional disease 598 prevention and control. Scientific reports, 7, p.43932.

599 Mweu, M.M., Fournié, G., Halasa, T., Toft, N., Nielsen, S.S., 2013. Temporal characterisation of the 600 network of Danish cattle movements and its implication for disease control. Prev. Vet. Med. 110, 601379.

602 Natale, F., Giovannini, A., Savini, L., Palma, D., Possenti, L., Fiore, G., Calistri, P., 2009. Network 603 analysis of Italian cattle trade patterns and evaluation of risks for potential disease spread. Prev. Vet. $604 \quad$ Med. 92, 341-350.

605 Ortiz-Pelaez, A., Pfeiffer, D.U., Soares-Magalhães, R.J., Guitian, F.J., 2006. Use of social network 606 analysis to characterize the pattern of animal movements in the initial phases of the 2001 foot and 607 mouth disease (FMD) epidemic in the UK. Prev. Vet. Med. 76, 40-55.

608 Palisson, A., Courcoul, A., Durand, B., 2016. Role of cattle movements in bovine tuberculosis spread 609 in France between 2005 and 2014. PLOS ONE 11, e0152578.

610 R Core Team (2019). R: A language and environment for statistical computing. R Foundation for 611 Statistical Computing, Vienna, Austria. URL https://www.R-project.org/.

612 Rautureau, S., Dufour, B., Durand, B., 2011. Vulnerability of animal trade networks to the spread of 613 infectious diseases: A methodological approach applied to evaluation and emergency control 614 strategies in cattle, France, 2005. Transboundary and emerging diseases 58(2), 110-120.

615 Ribeiro-Lima, J., Enns, E.A., Thompson, B., Craft, M.E., Wells, S.J., 2015. From network analysis to risk 616analysis--an approach to risk-based surveillance for bovine tuberculosis in Minnesota, US. Prev. Vet. 617 Med. 118.

618 Robinson, S.E., Christley, R.M., 2007. Exploring the role of auction markets in cattle movements 619 within Great Britain. Prev. Vet. Med. 81, 21. 
620 VanderWaal, K., Enns, E.A., Picasso, C., Alvarez, J., Perez, A., Fernandez, F., Gil, A., Craft, M., Wells, S., 621 2017. Optimal surveillance strategies for bovine tuberculosis in a low-prevalence country. Scientific 622 Reports, 7.

623 VanderWaal, K.L., Picasso, C., Enns, E.A., Craft, M.E., Alvarez, J., Fernandez, F., Gil, A., Perez, A., 624 Wells, S., 2016. Network analysis of cattle movements in Uruguay: Quantifying heterogeneity for 625 risk-based disease surveillance and control. Preventive veterinary medicine JID - 8217463 $123,12-$

62622.

627 Vernon, M.C., Keeling, M.J., 2009. Representing the UK's cattle herd as static and dynamic networks. 628 Proc Biol Sci 276, 469.

629 Vidondo, B., Voekl, B., 2018. Dynamic network measures reveal the impact of cattle markets and 630 alpine summering on the risk of epidemic outbreaks in the Swiss cattle population. BMC Veterinary 631 Research 14, 88.

632 Watts, D.J., 1999. Small Worlds: The Dynamics of Networks between Order and Randomness.

633 Princeton University Press, Princeton, N.J.

634 Wickham, H., 2009. Ggplot2: Elegant Graphics for Data Analysis. Springer-Verlag, New York. 


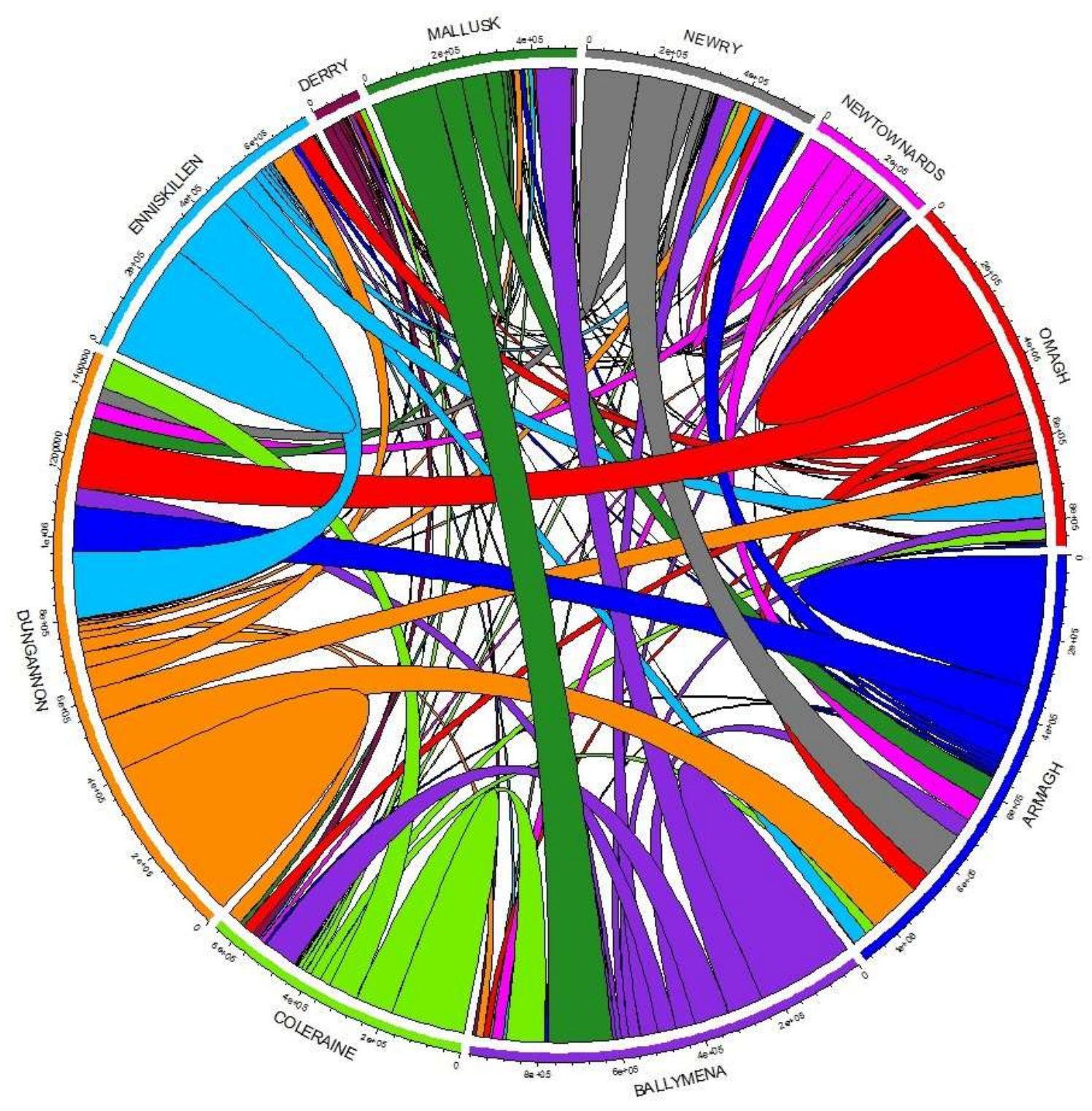




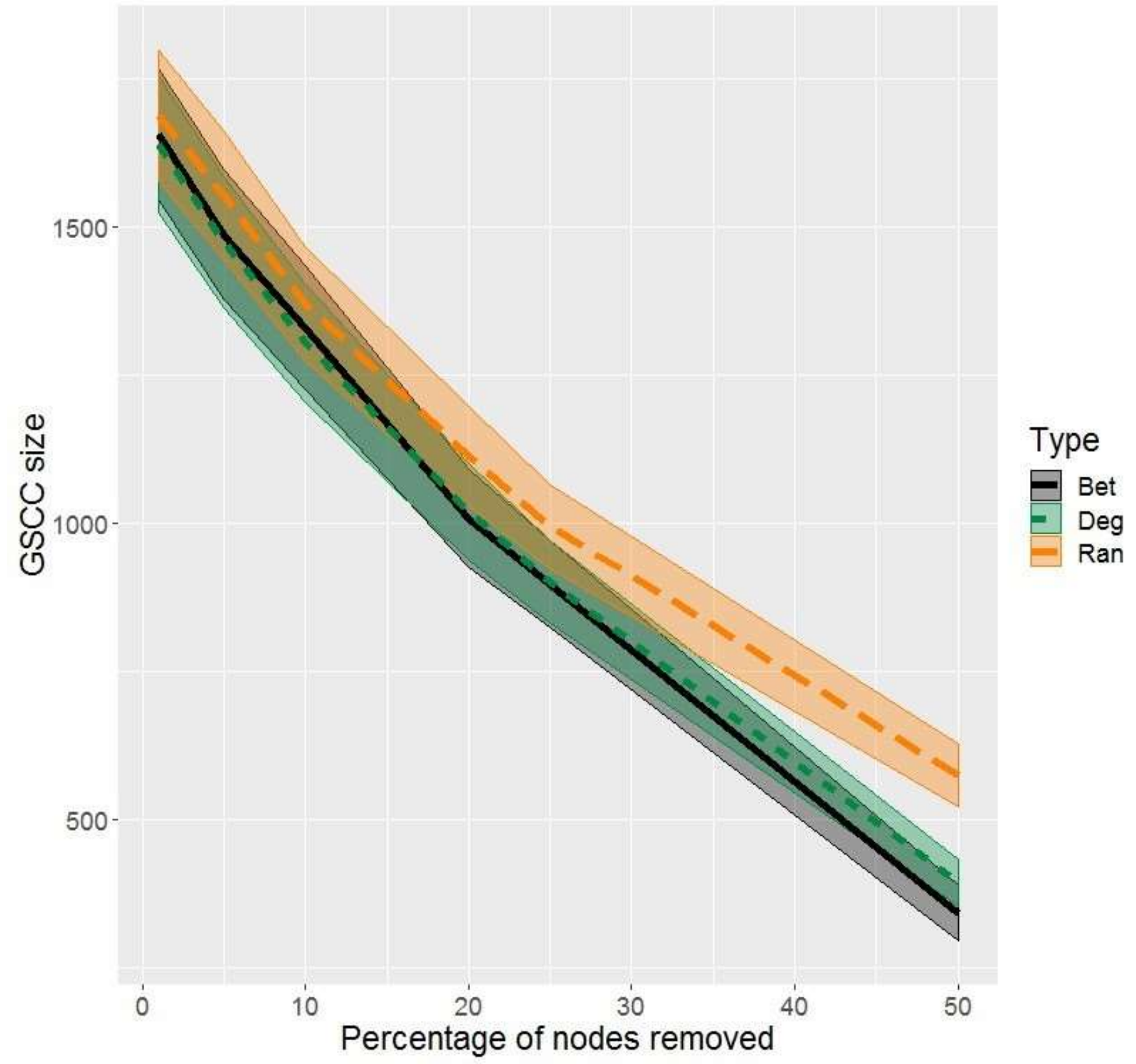




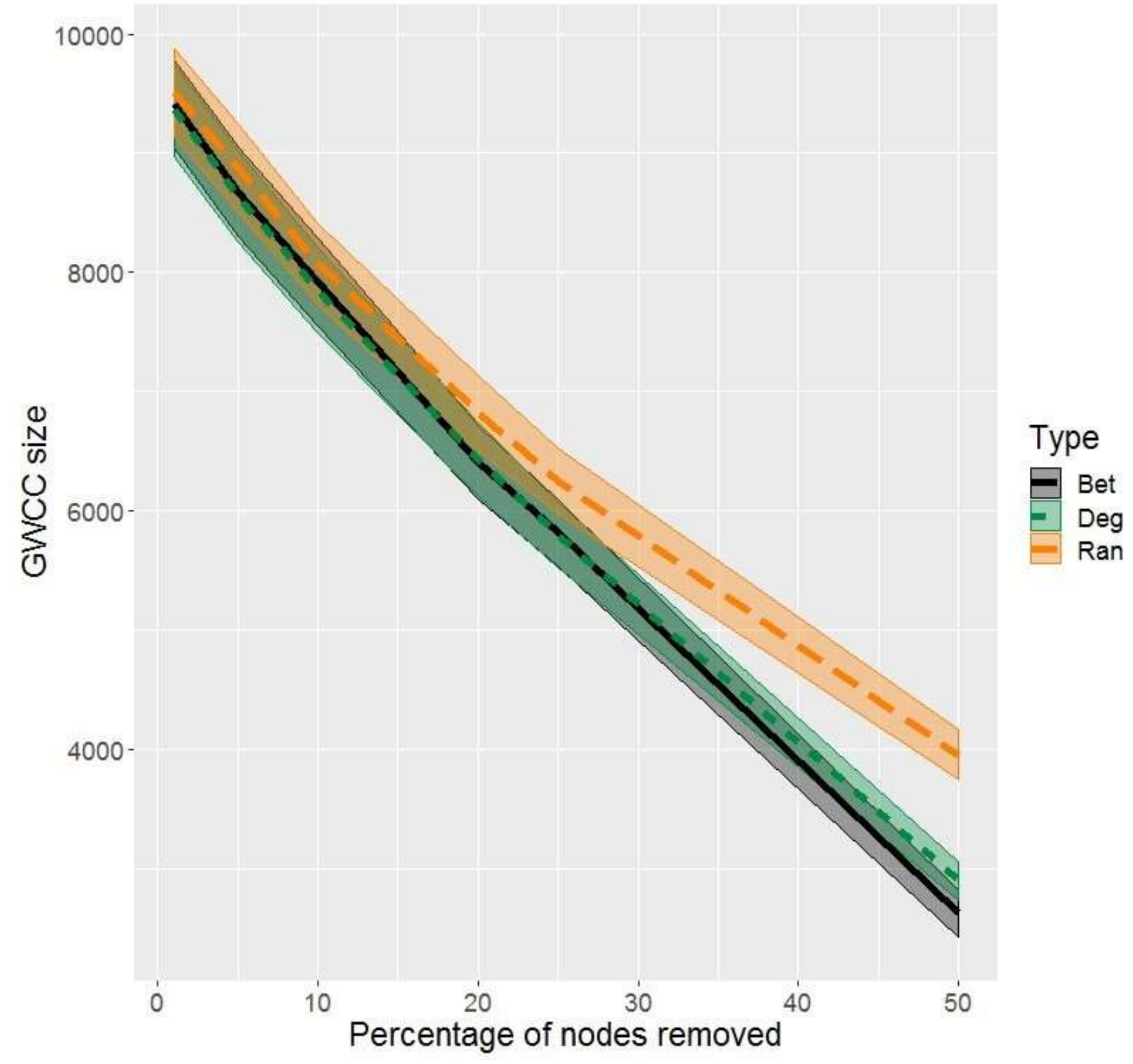




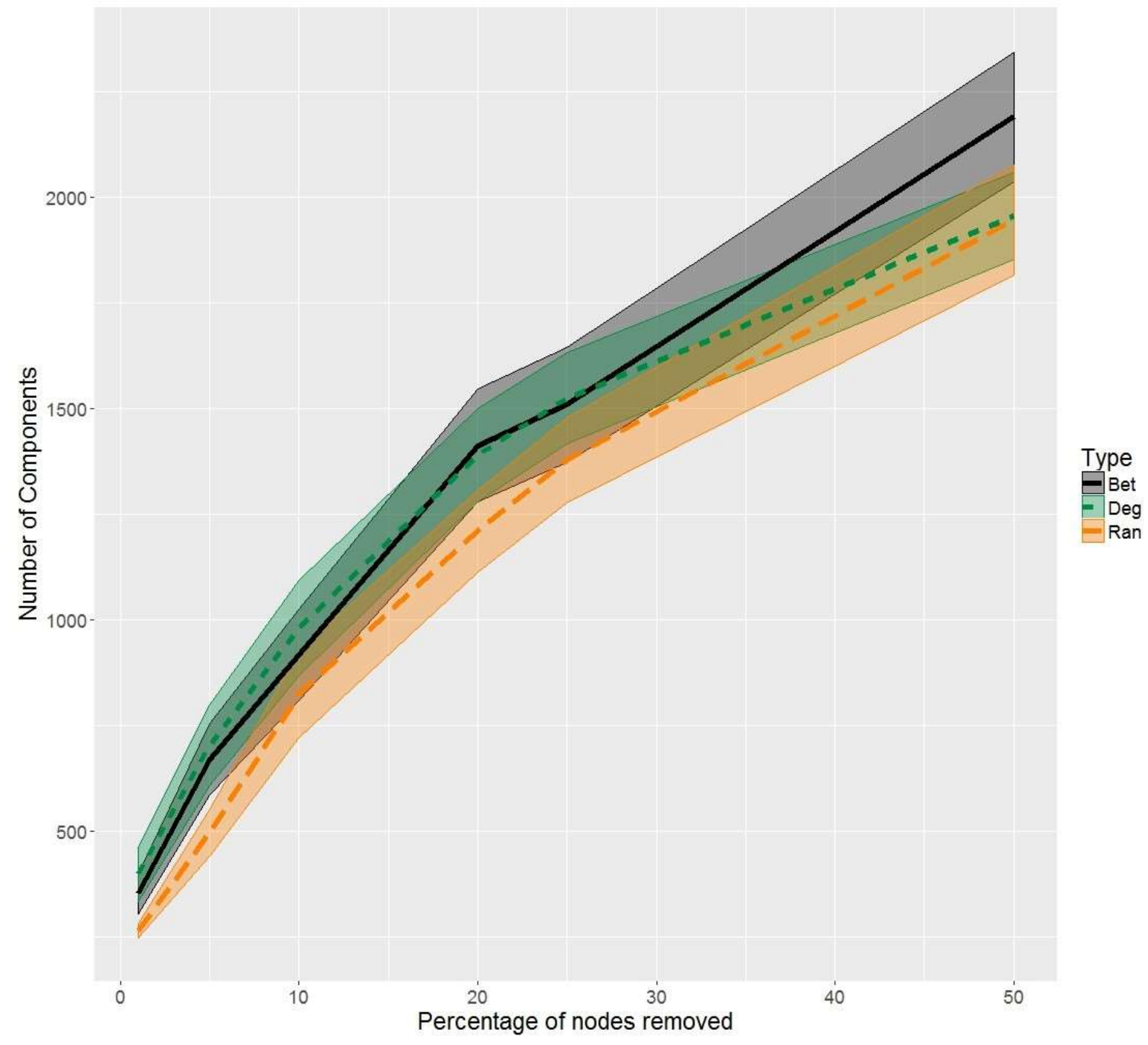


Fig. 1: Chord diagram presenting cattle movements occurring within and between Northern Ireland's DVOs (District Veterinary Office of DAERA) during 2010-2015. A map of each DVO location is provided in Fig S15 of the Supplementary Material.

Fig. 2: Mean (lines: Black and Solid = Betweenness (Bet) based node removal, Green and Dashed = Degree (Deg) based node removal, Orange and Long-dashed = Random (Ran) node removal) and $95 \% \mathrm{Cl}$ (coloured ribbons: Black $=$ Betweenness based node removal, Green $=$ Degree based node removal, Orange $=$ Random node removal) GSCC size for the networks over 2010-2015 and stratified by node removal.

Fig. 3: Mean (lines: Black and Solid = Betweenness (Bet) based node removal, Green and Dashed = Degree (Deg) based node removal, Orange and Long-dashed = Random (Ran) node removal) and 95\% $\mathrm{Cl}$ (coloured ribbons: Black = Betweenness based node removal, $\mathrm{Green}=$ Degree based node removal, Orange $=$ Random node removal) GWCC size for the networks over 2010-2015 and stratified by node removal.

Fig. 4: Mean (lines: Black and Solid = Betweenness (Bet) based node removal, Green and Dashed = Degree (Deg) based node removal, Orange and Long-dashed = Random (Ran) node removal) and $95 \% \mathrm{Cl}$ (coloured ribbons: Black $=$ Betweenness based node removal, Green = Degree based node removal, Orange $=$ Random node removal) number of components for the networks over 2010-2015 and stratified by node removal. 


\section{Supplementary Material}

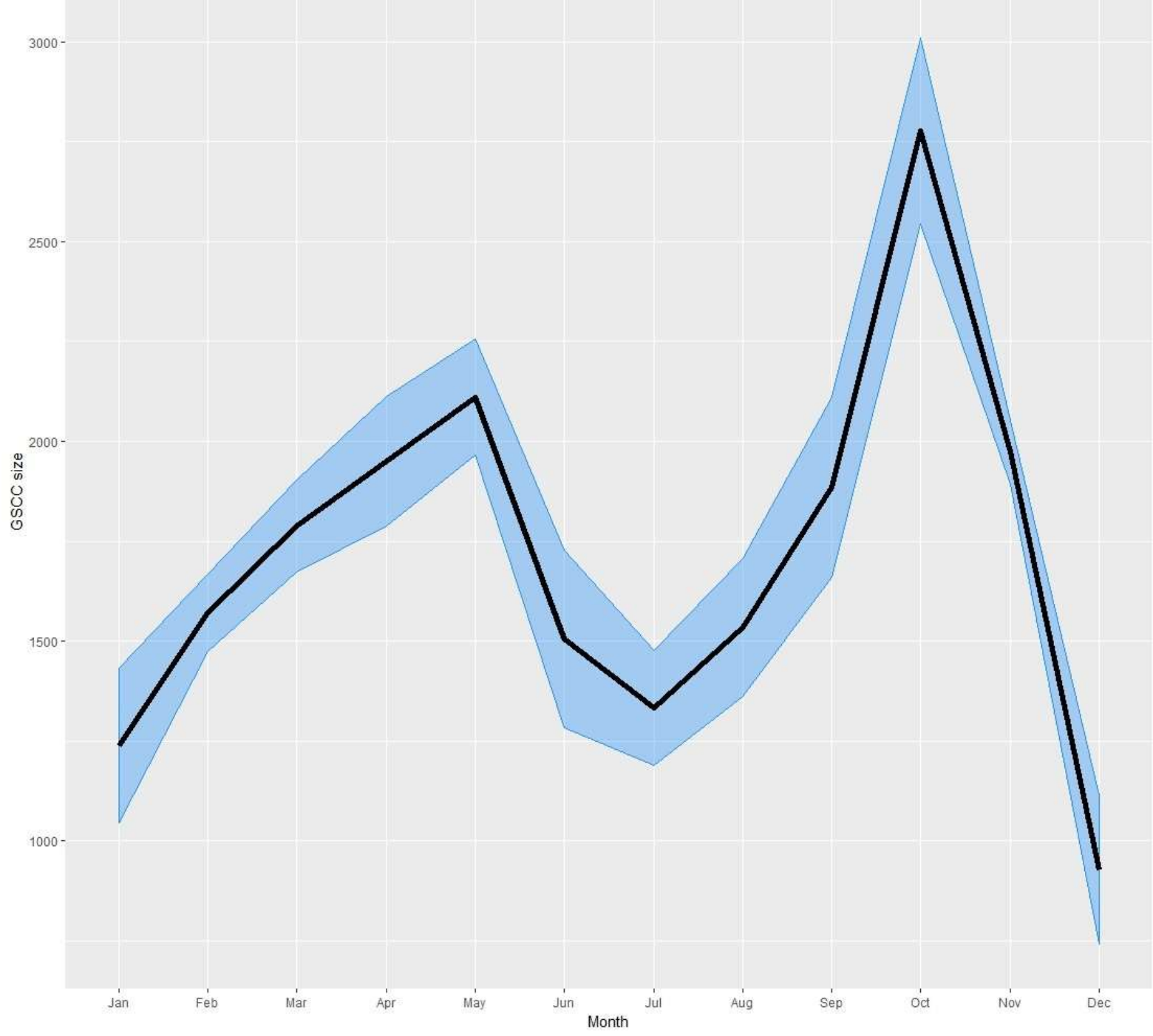

S1: Mean size (black line) and $95 \% \mathrm{Cl}$ (blue ribbon) of the GSCC across the year for the study period 2010-2015. 


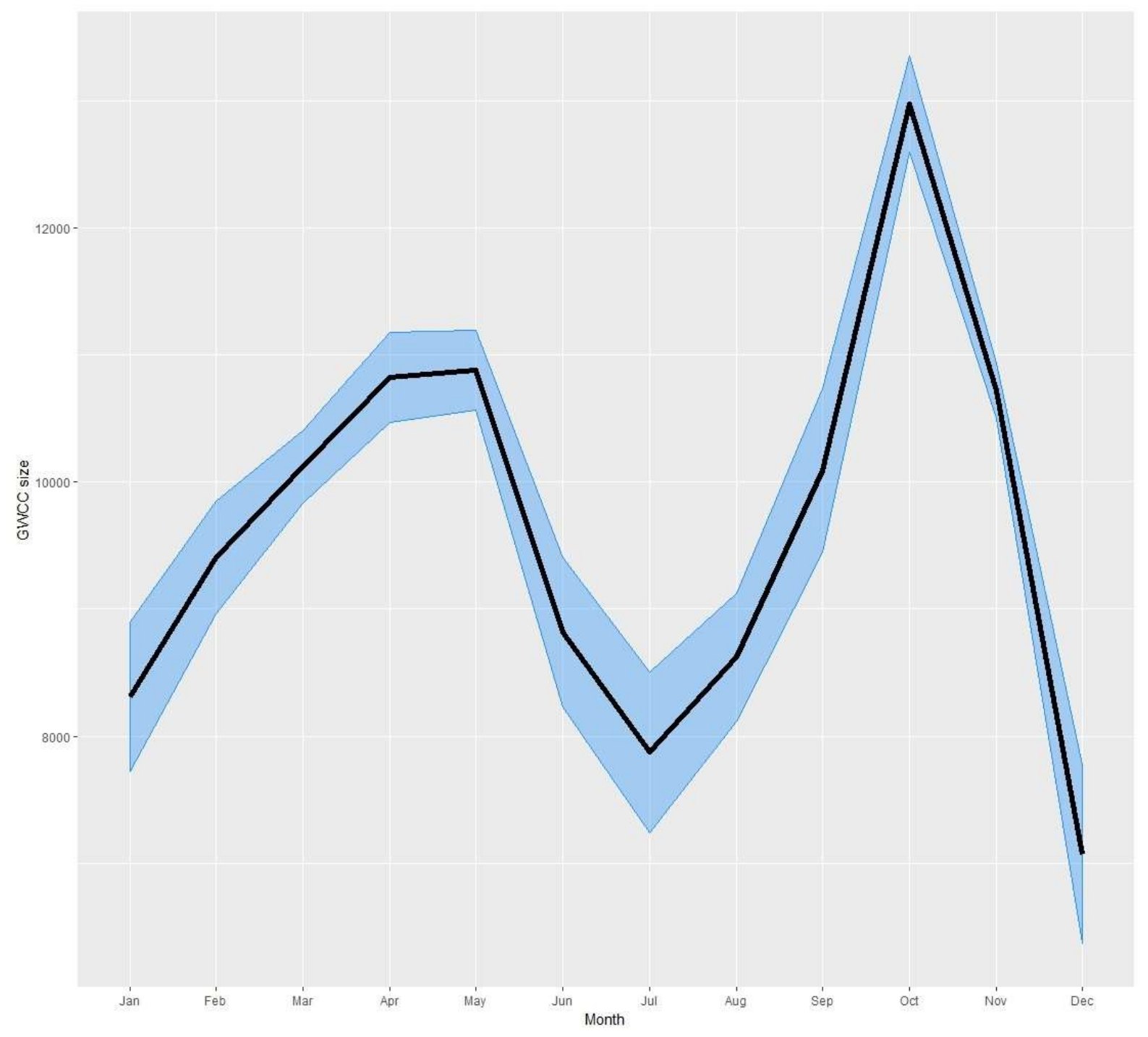

S2: Mean size (black line) and $95 \% \mathrm{Cl}$ (blue ribbon) of the GWCC across the year for the study period 2010-2015. 


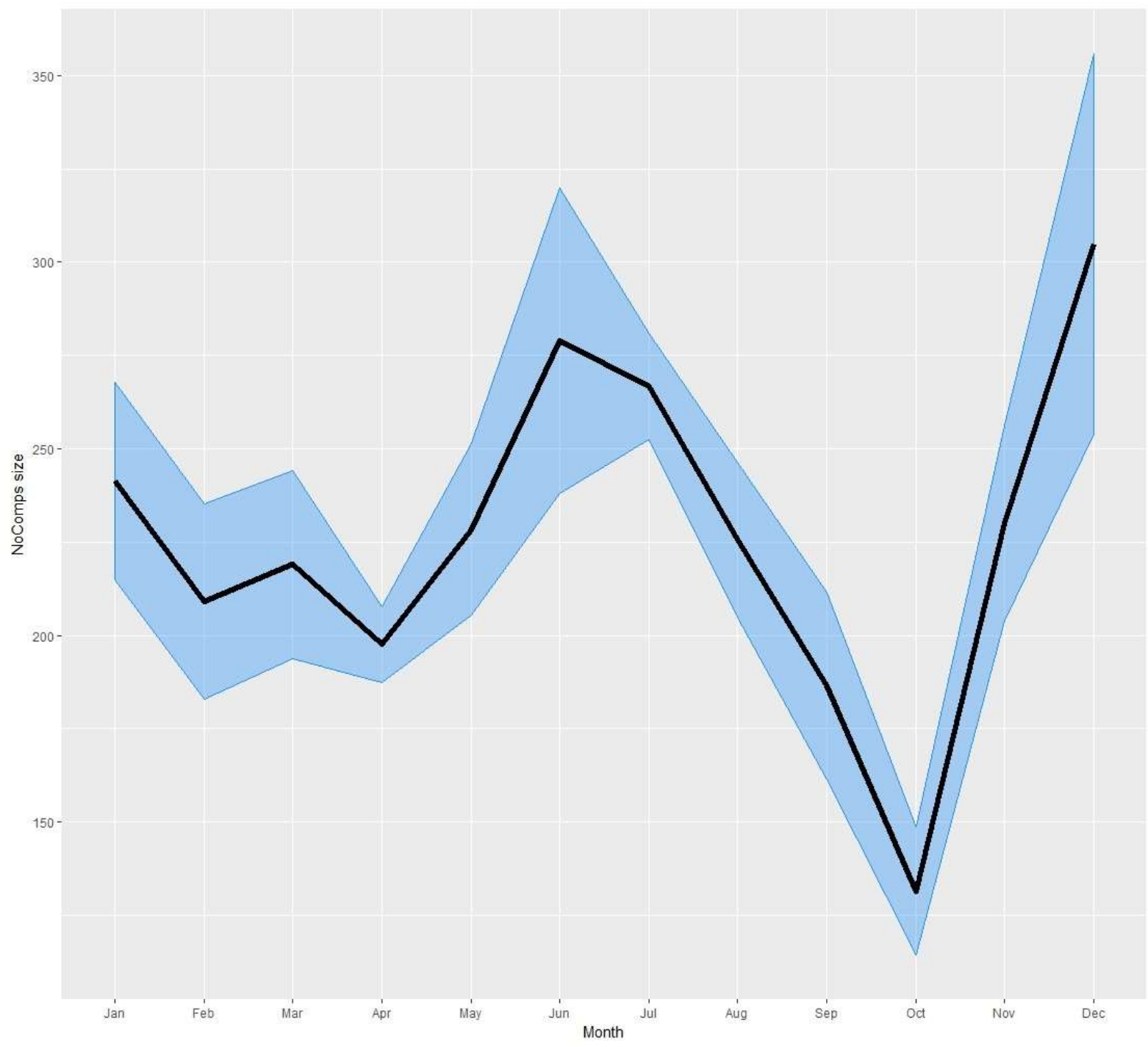

S3: Mean size (black line) and $95 \% \mathrm{Cl}$ (blue ribbon) of the number of components across the year for the study period 2010-2015. 


$$
W
$$




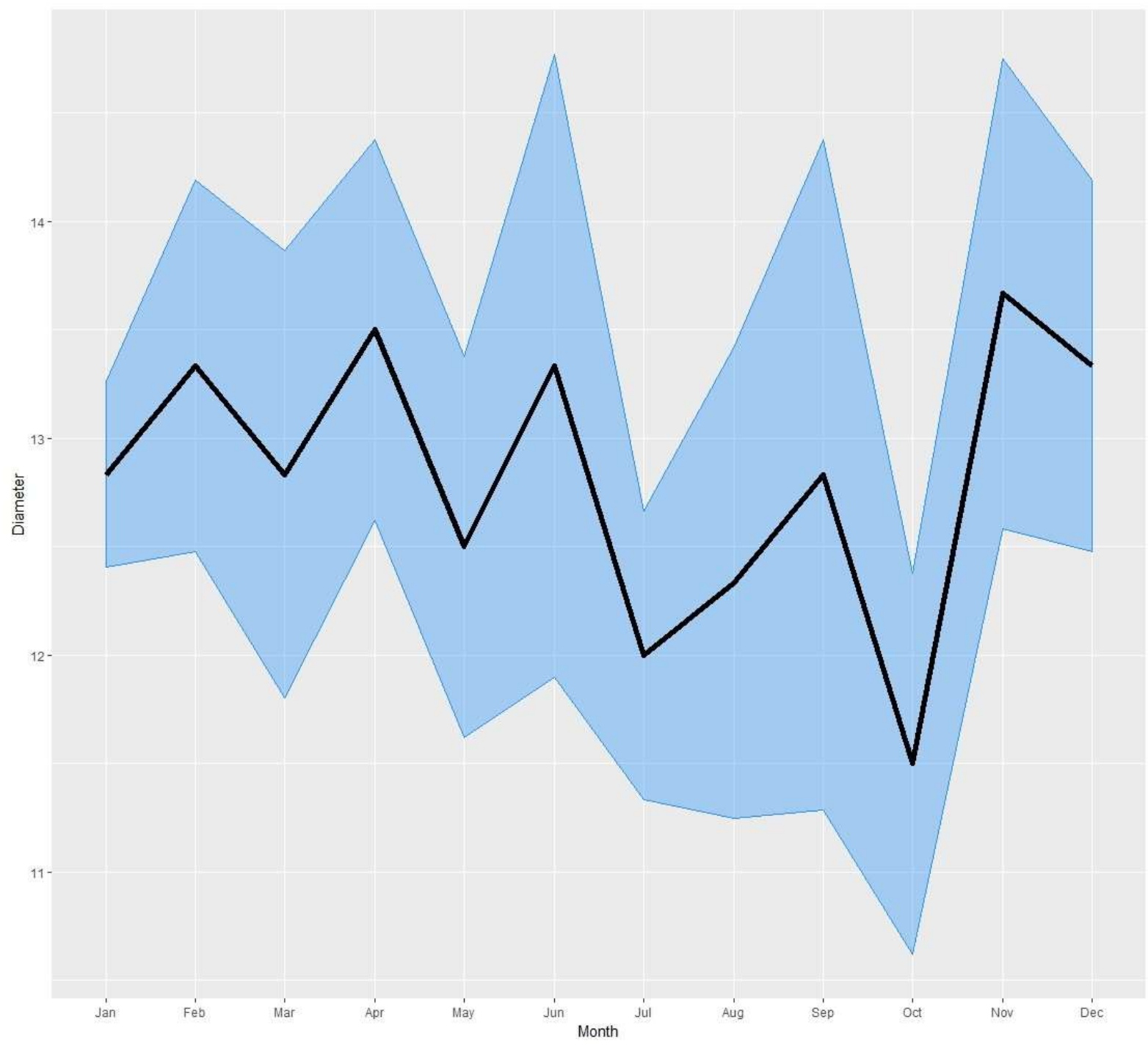

S5: Mean size (black line) and $95 \% \mathrm{Cl}$ (blue ribbon) of the diameter across the year for the study period 2010-2015. 


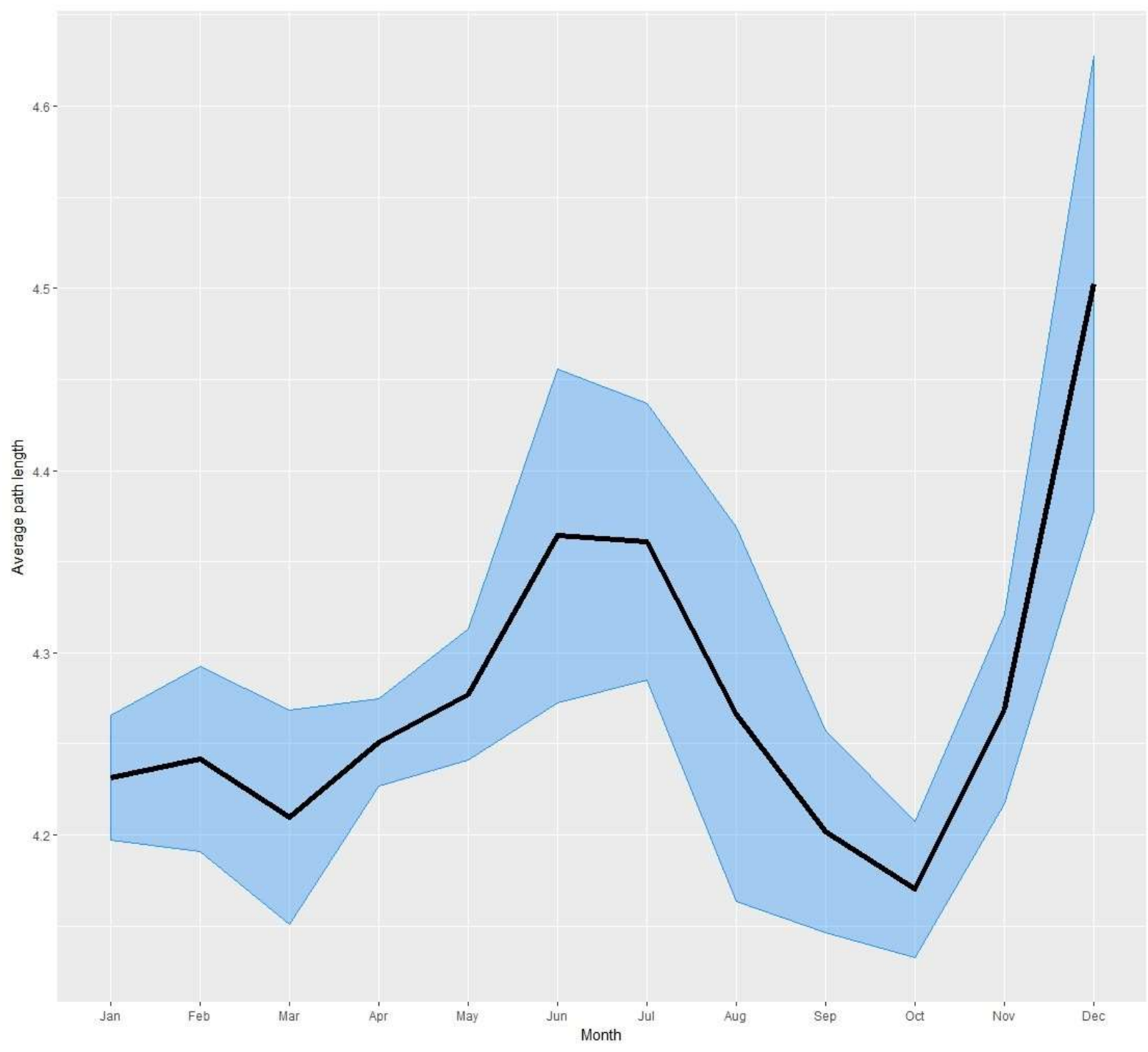

S6: Mean size (black line) and $95 \% \mathrm{Cl}$ (blue ribbon) of the average path length across the year for the study period 2010-2015. 


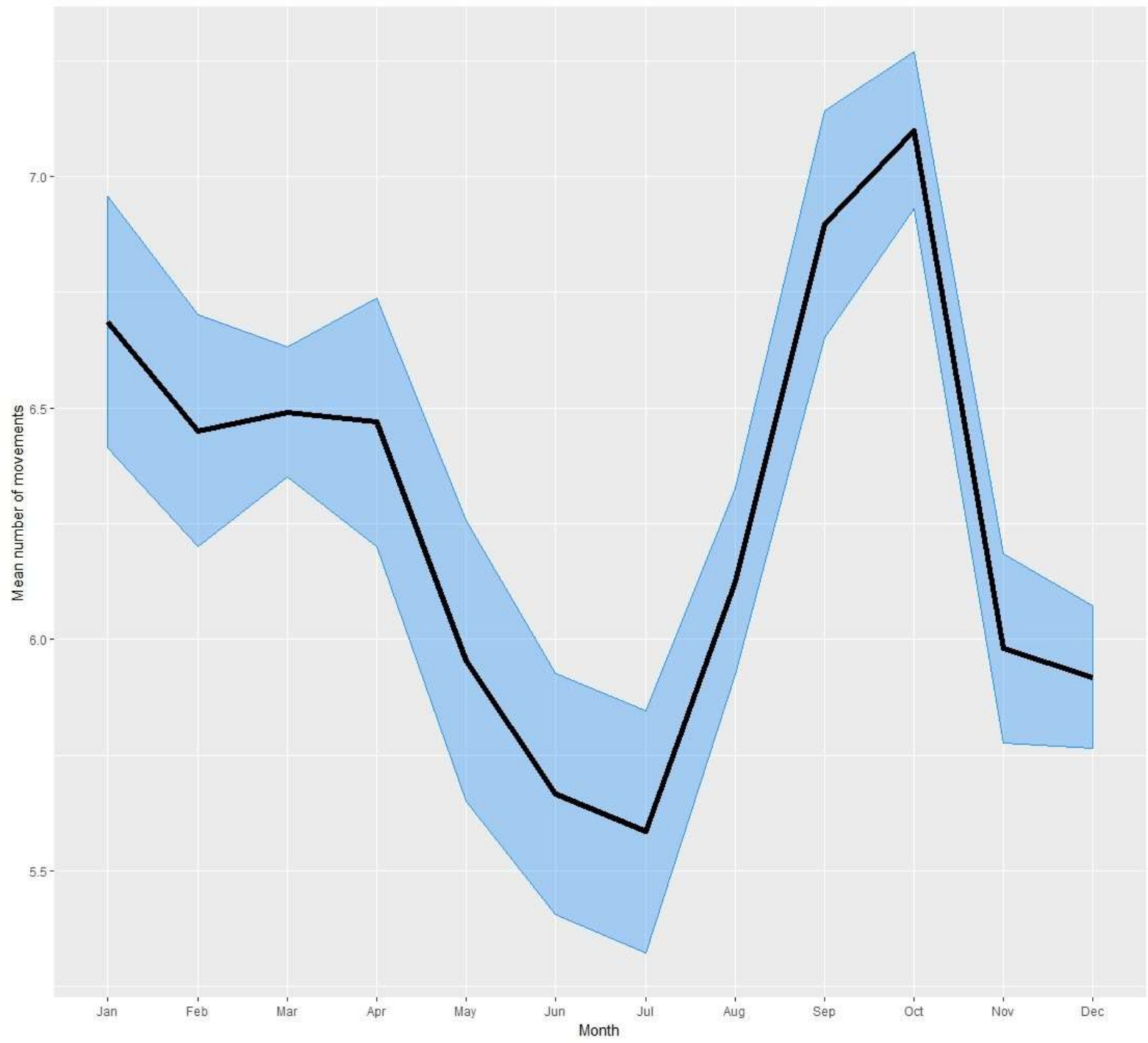

S7: Mean size (black line) and $95 \% \mathrm{Cl}$ (blue ribbon) of the mean number of movements between distinct node pairs across the year for the study period 2010-2015. 


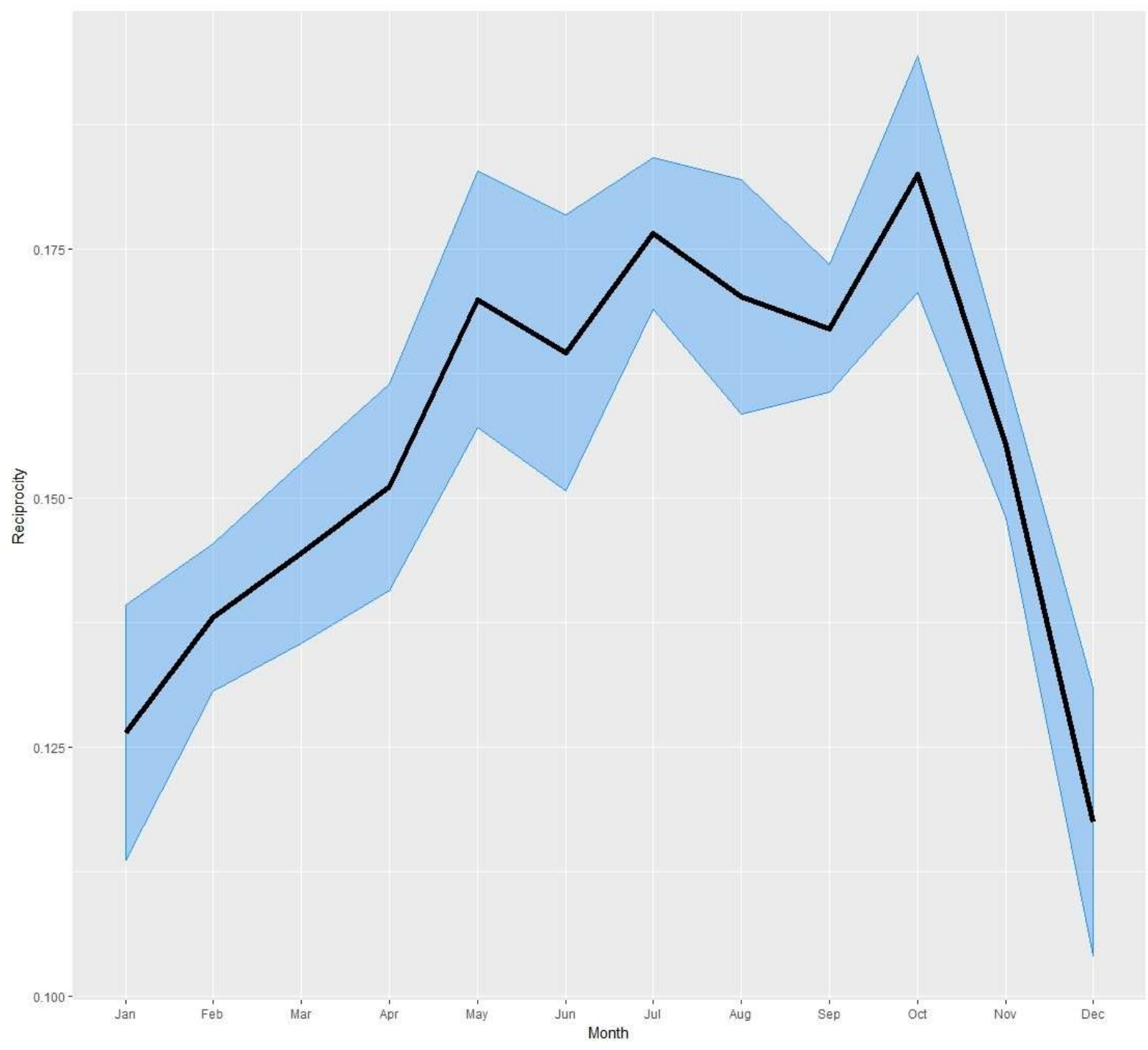

S8: Mean size (black line) and $95 \% \mathrm{Cl}$ (blue ribbon) of the reciprocity across the year for the study period 2010-2015. 


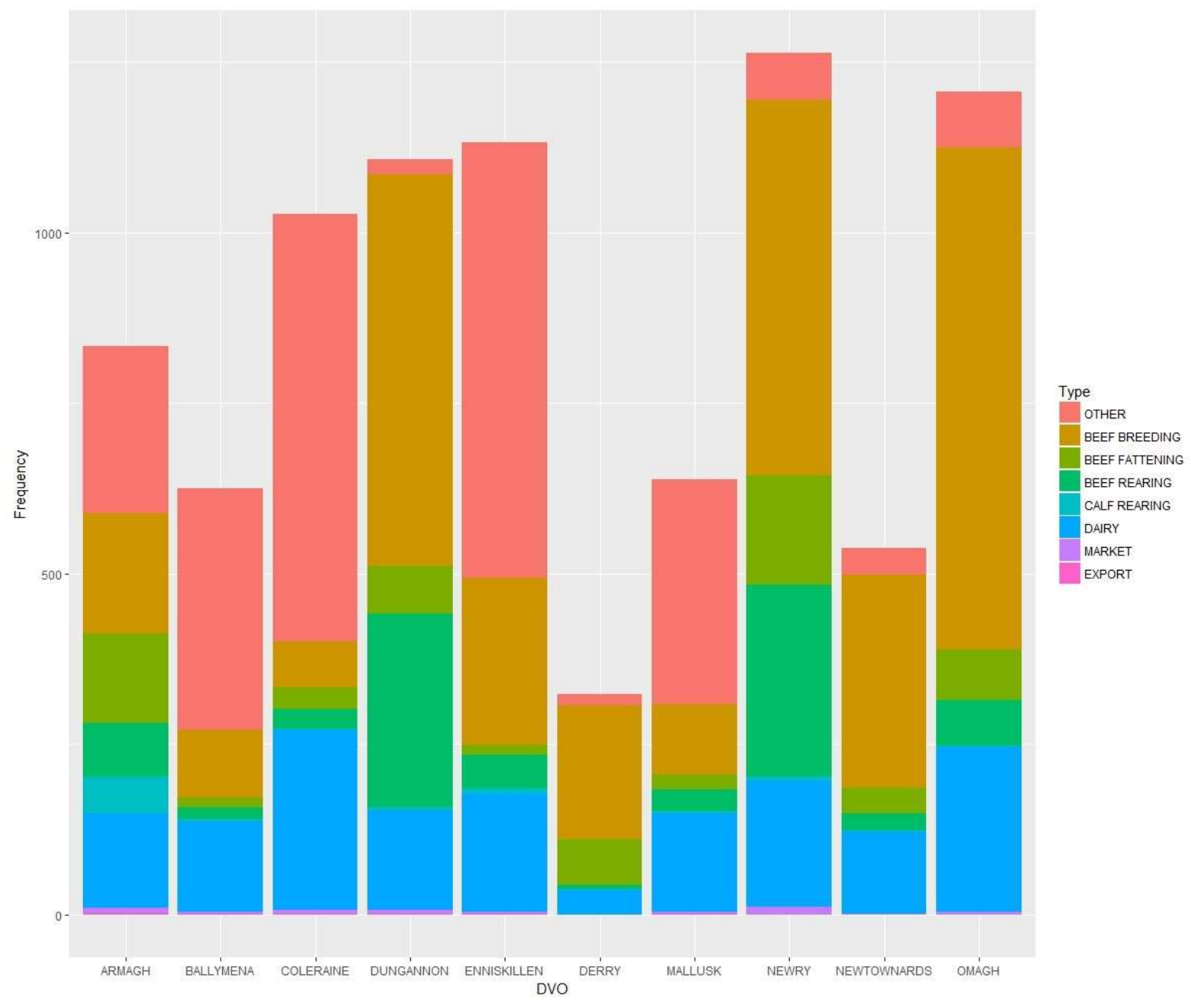

S9: Breakdown of the membership of the GSCC in 2010. 


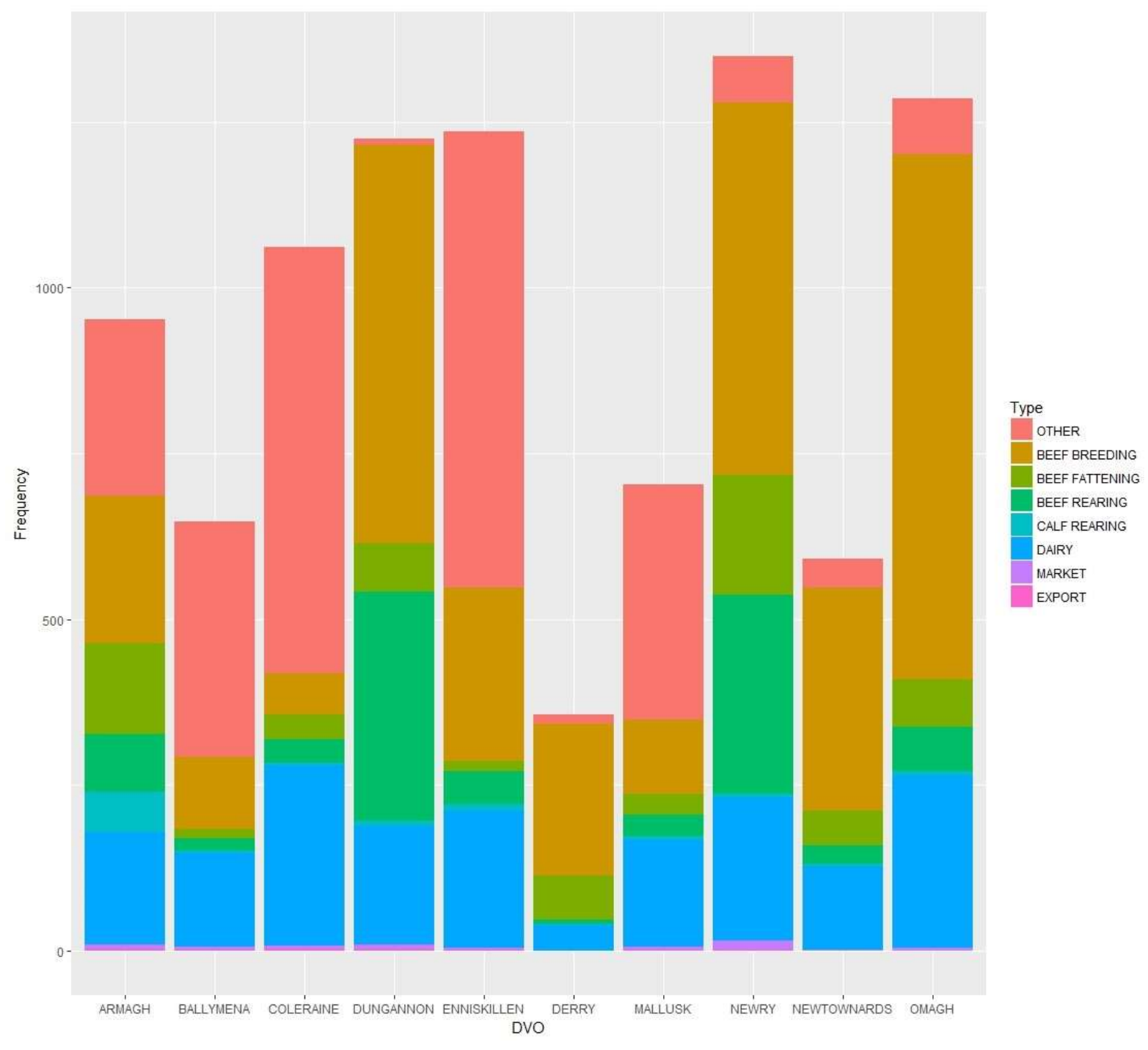

S10: Breakdown of the membership of the GSCC in 2011. 


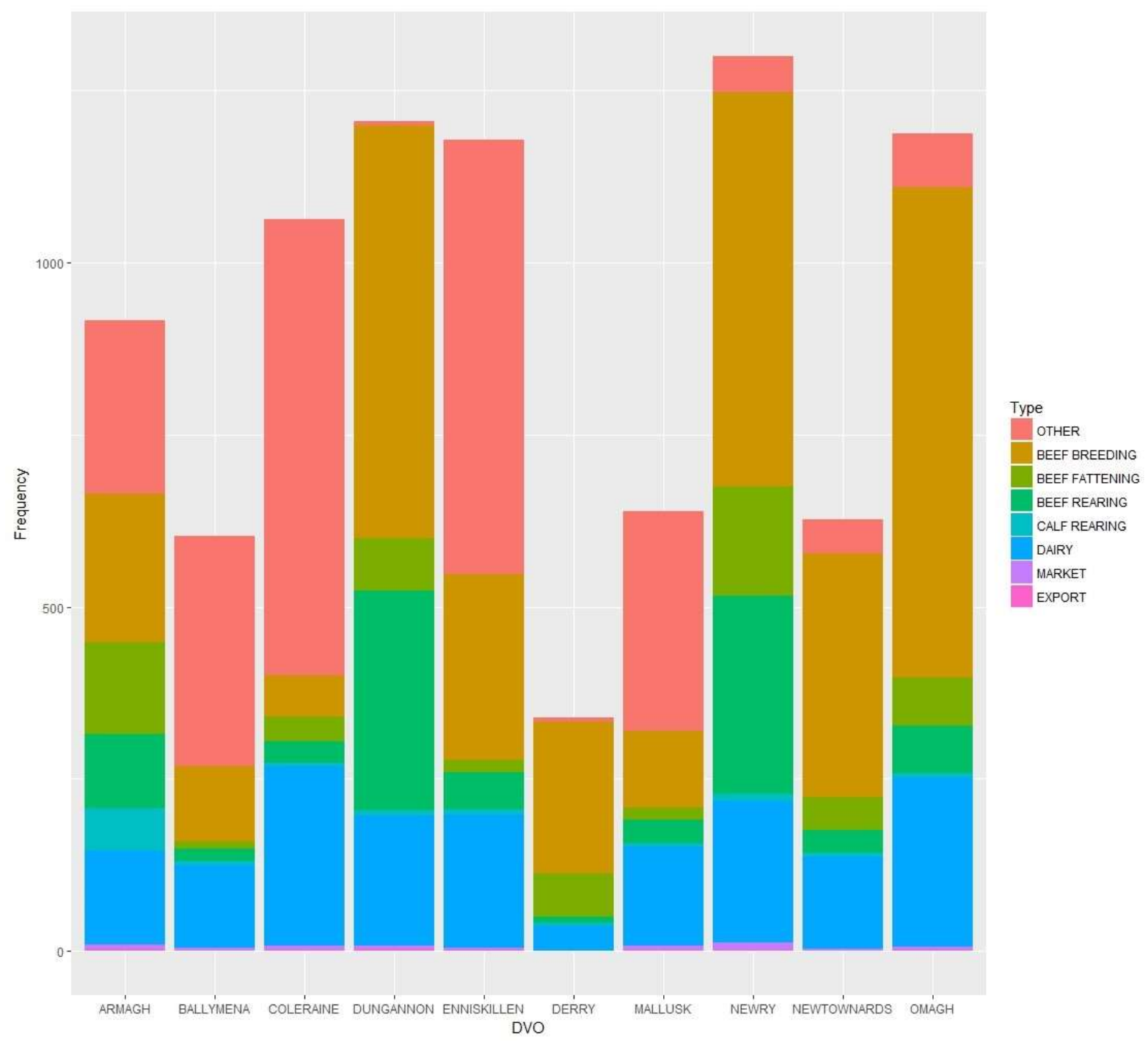

S11: Breakdown of the membership of the GSCC in 2012. 


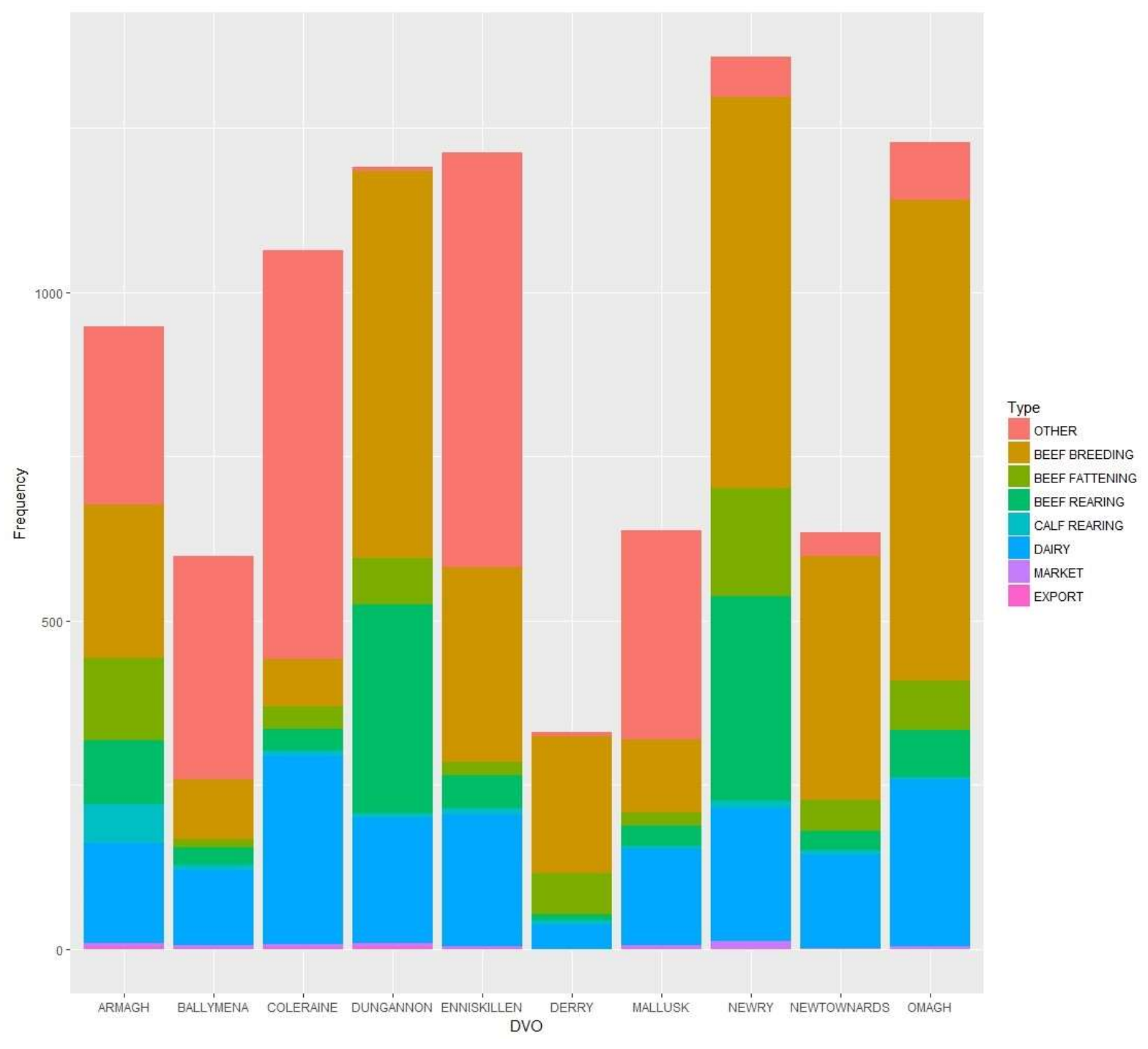

S12: Breakdown of the membership of the GSCC in 2013. 


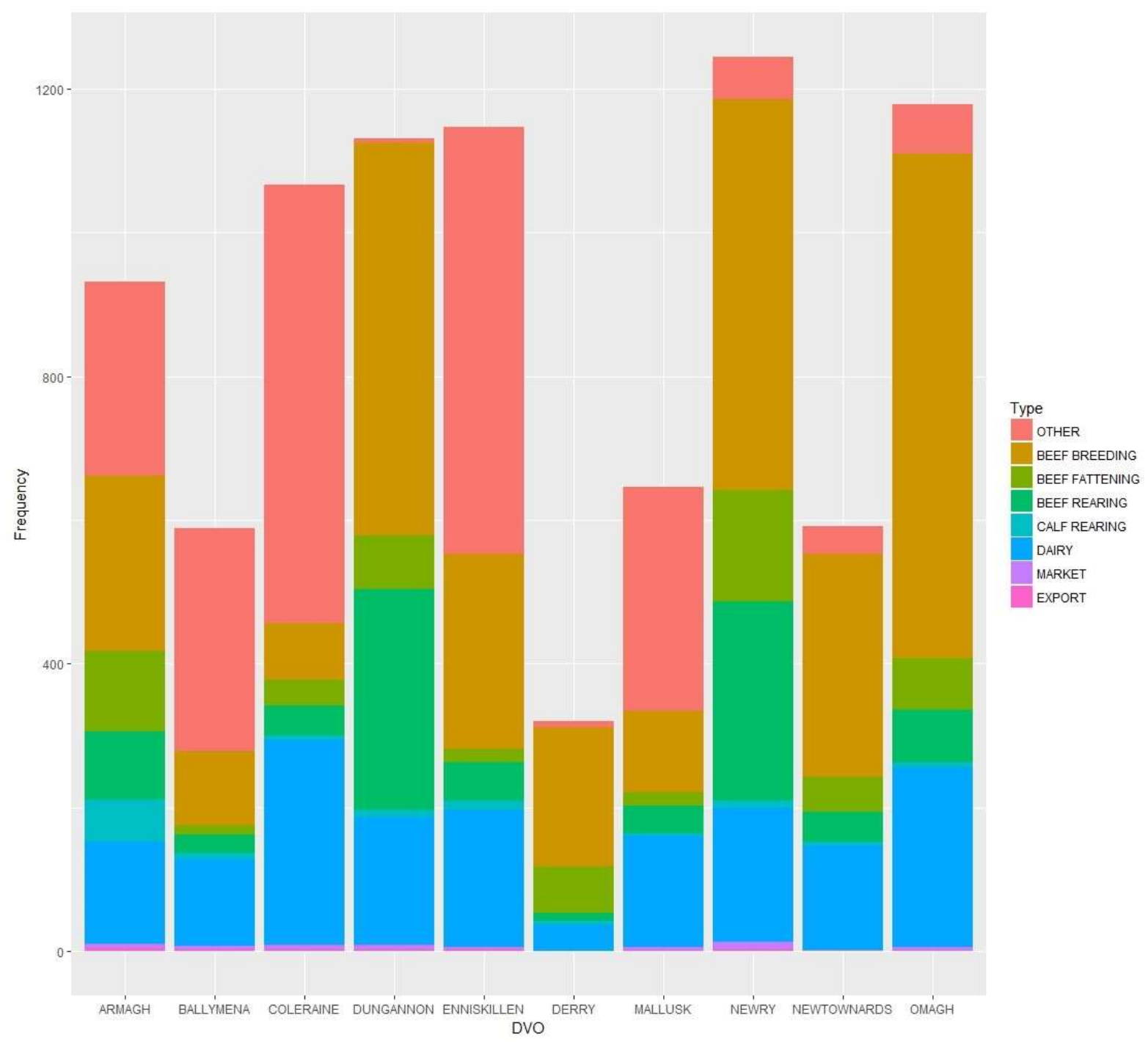

S13: Breakdown of the membership of the GSCC in 2014. 


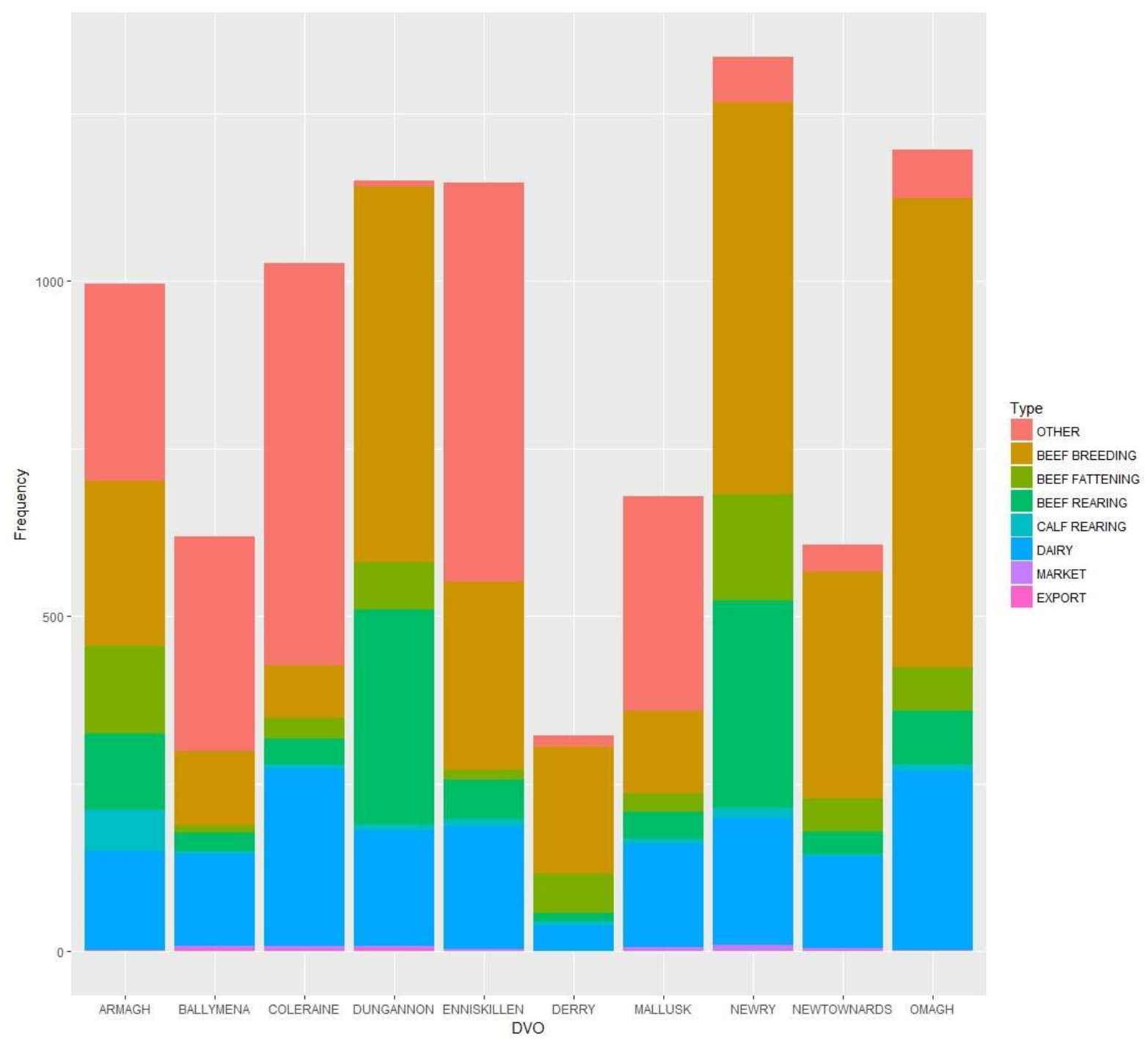

S14: Breakdown of the membership of the GSCC in 2015. 


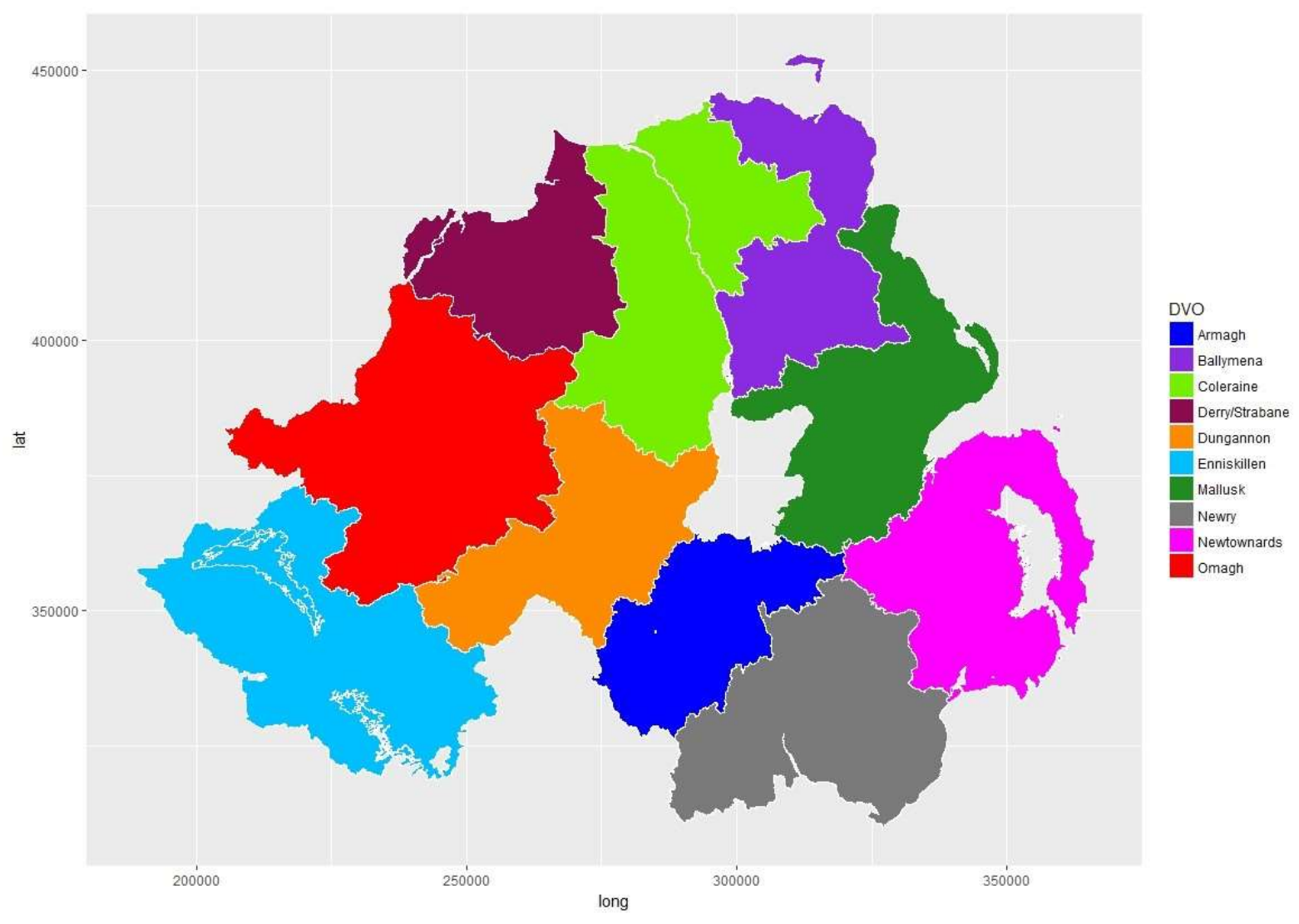

S15: Spatial representation of the 10 DVOs in Northern Ireland. 

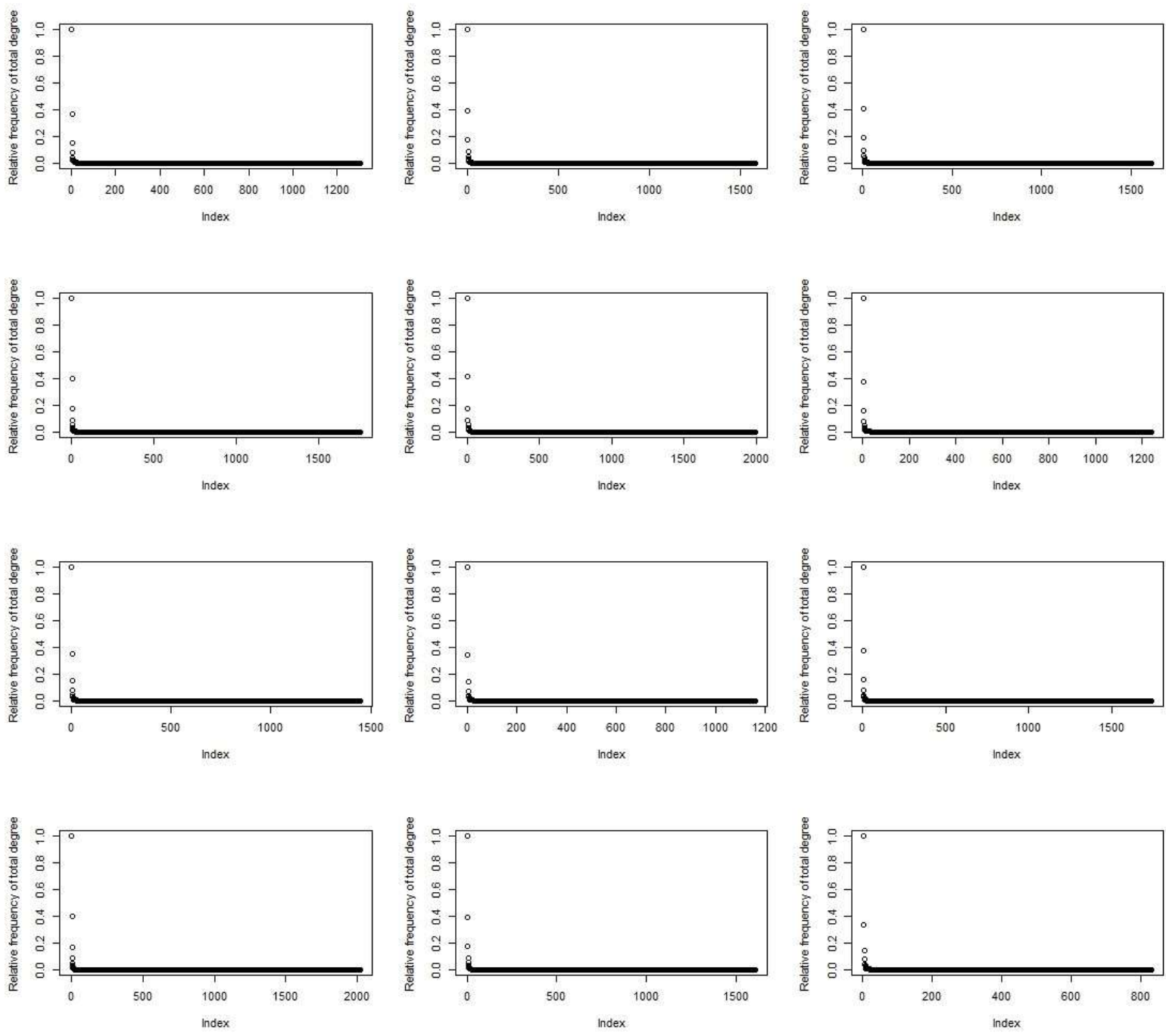

S16: Degree distribution of each network over 2010 (read left to right by row). 

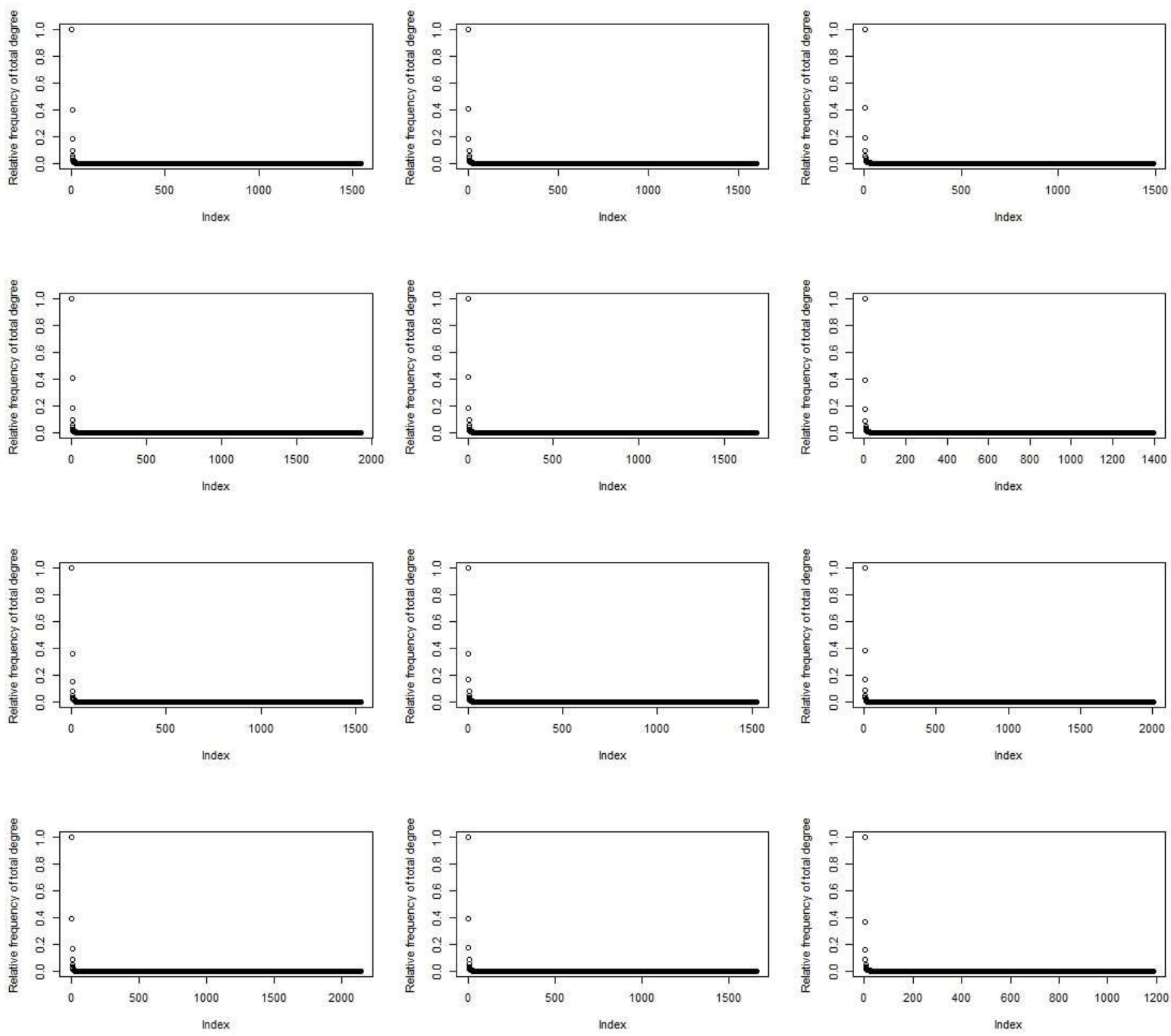

S17: Degree distribution of each network over 2011 (read left to right by row). 

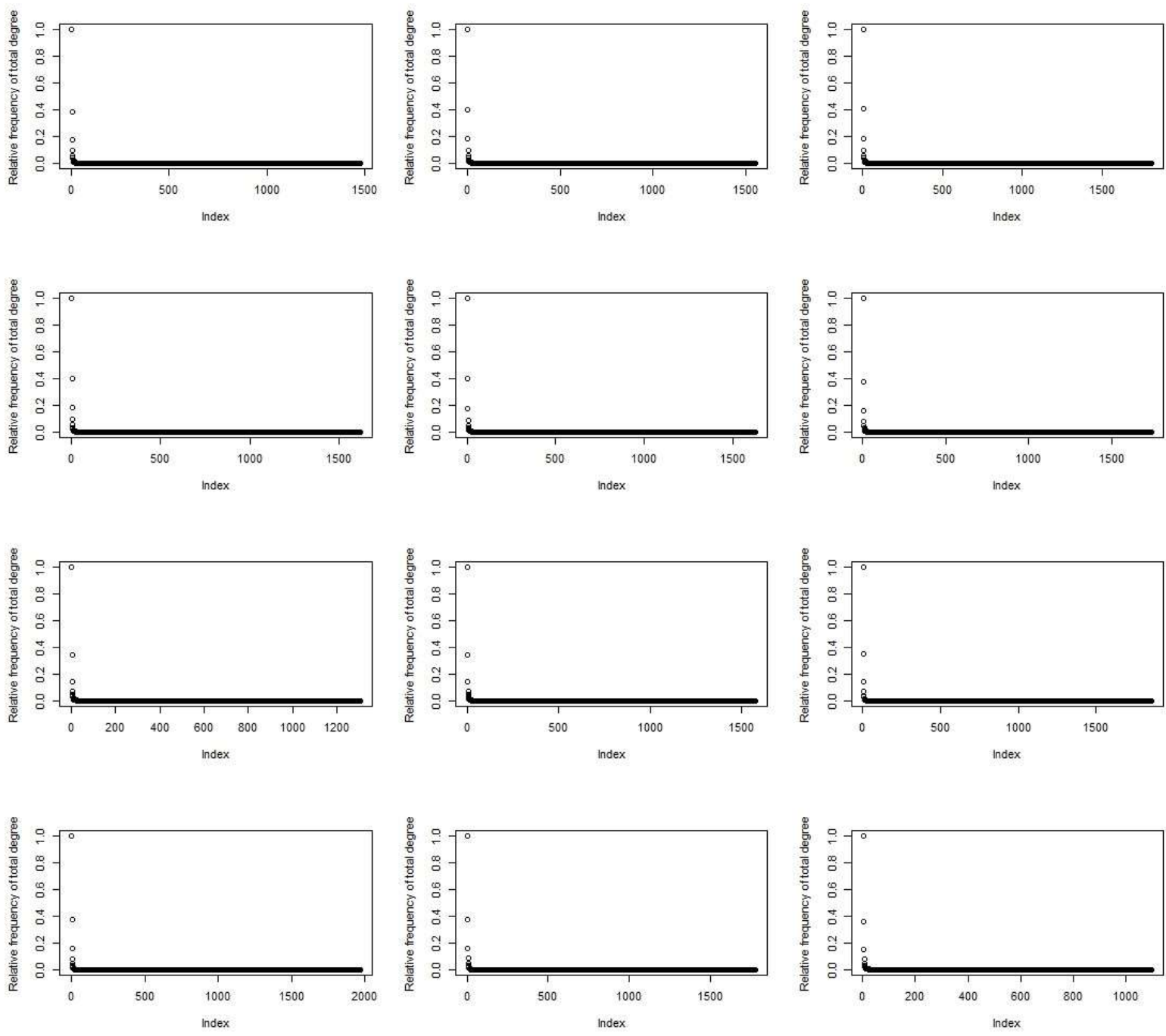

S18: Degree distribution of each network over 2012 (read left to right by row). 

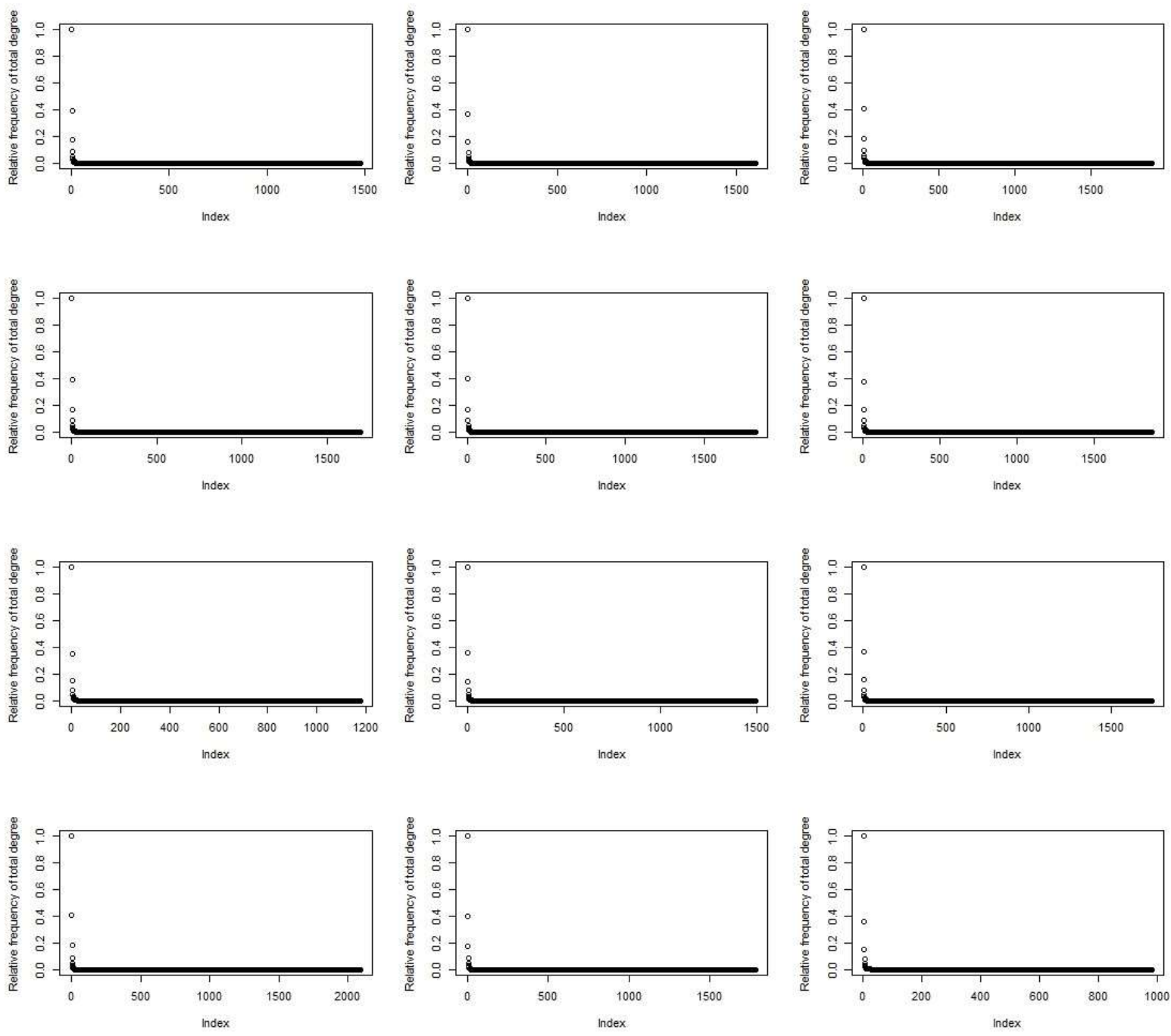

S19: Degree distribution of each network over 2013 (read left to right by row). 

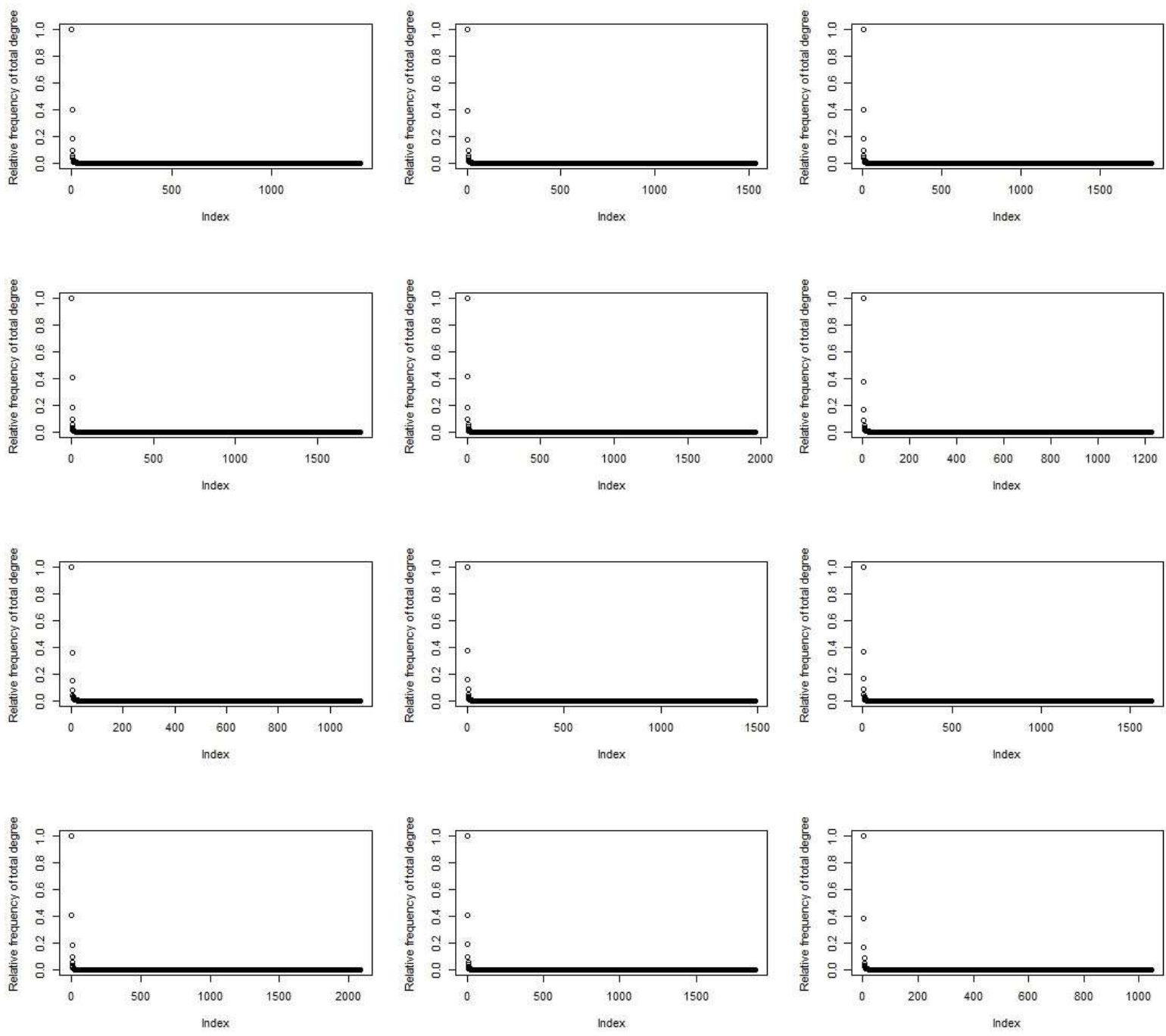

S20: Degree distribution of each network over 2014 (read left to right by row). 

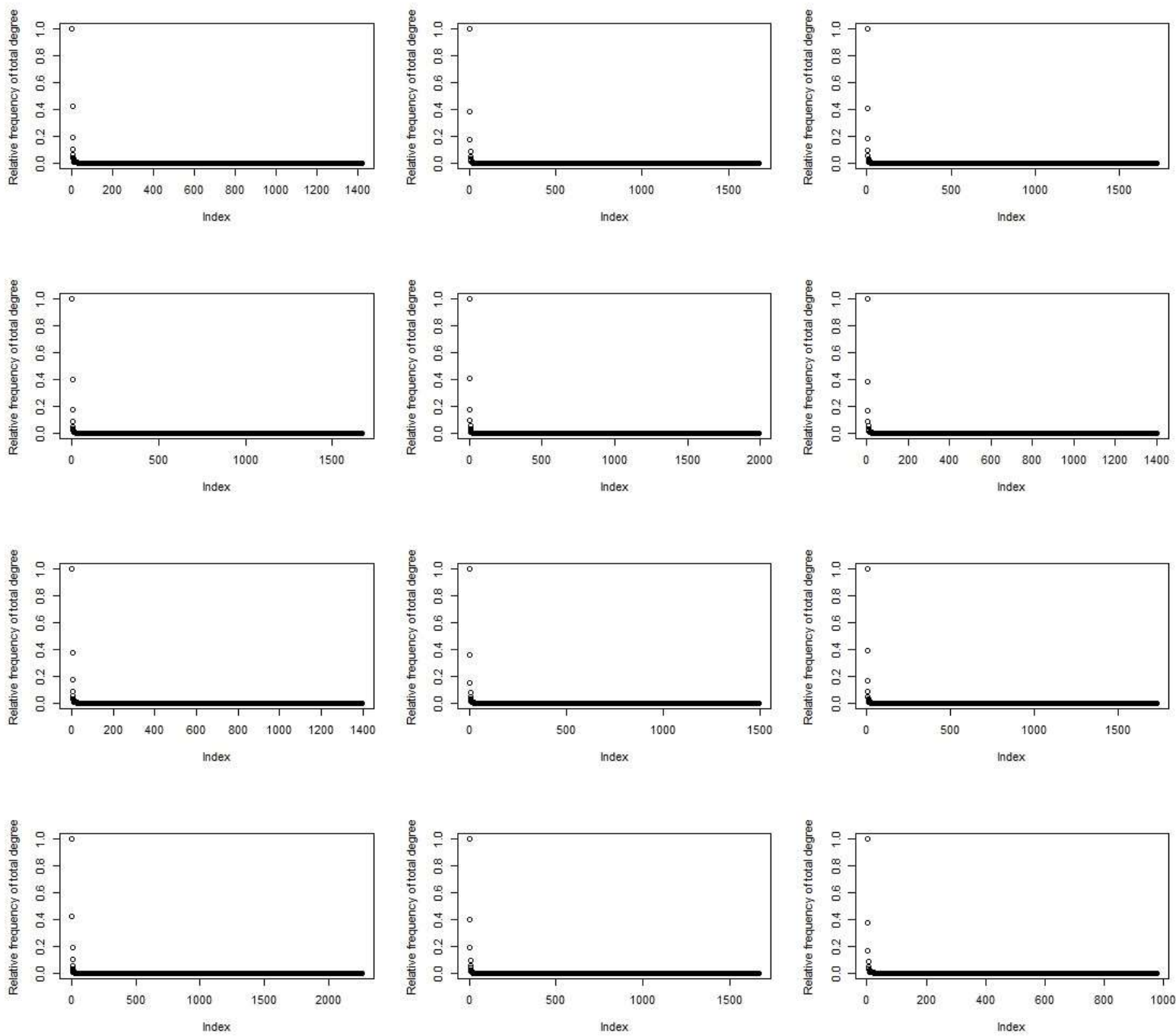

S21: Degree distribution of each network over 2015 (read left to right by row). 

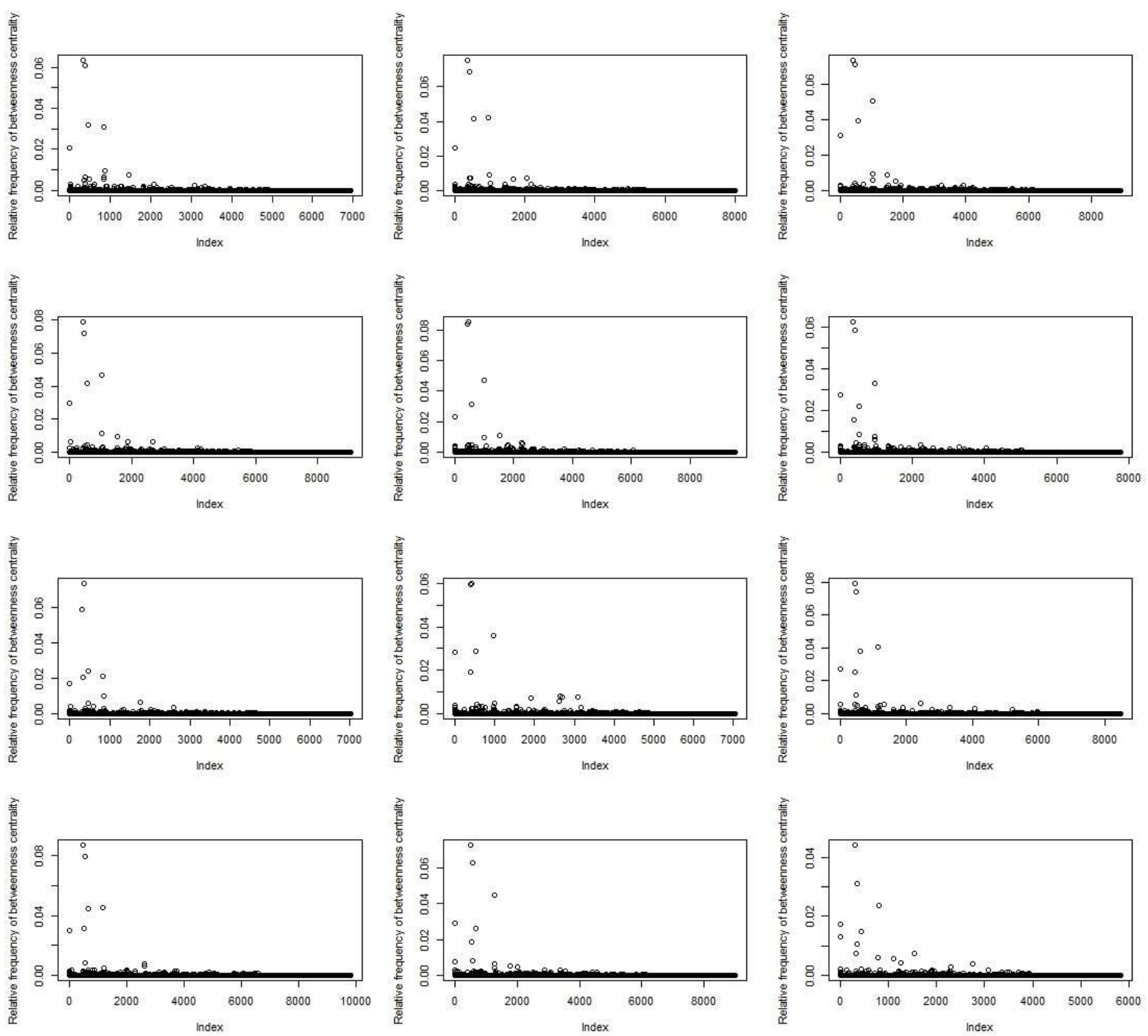

row). 
S22: Normalised betweenness centrality distribution of each network over 2010 (read left to right by
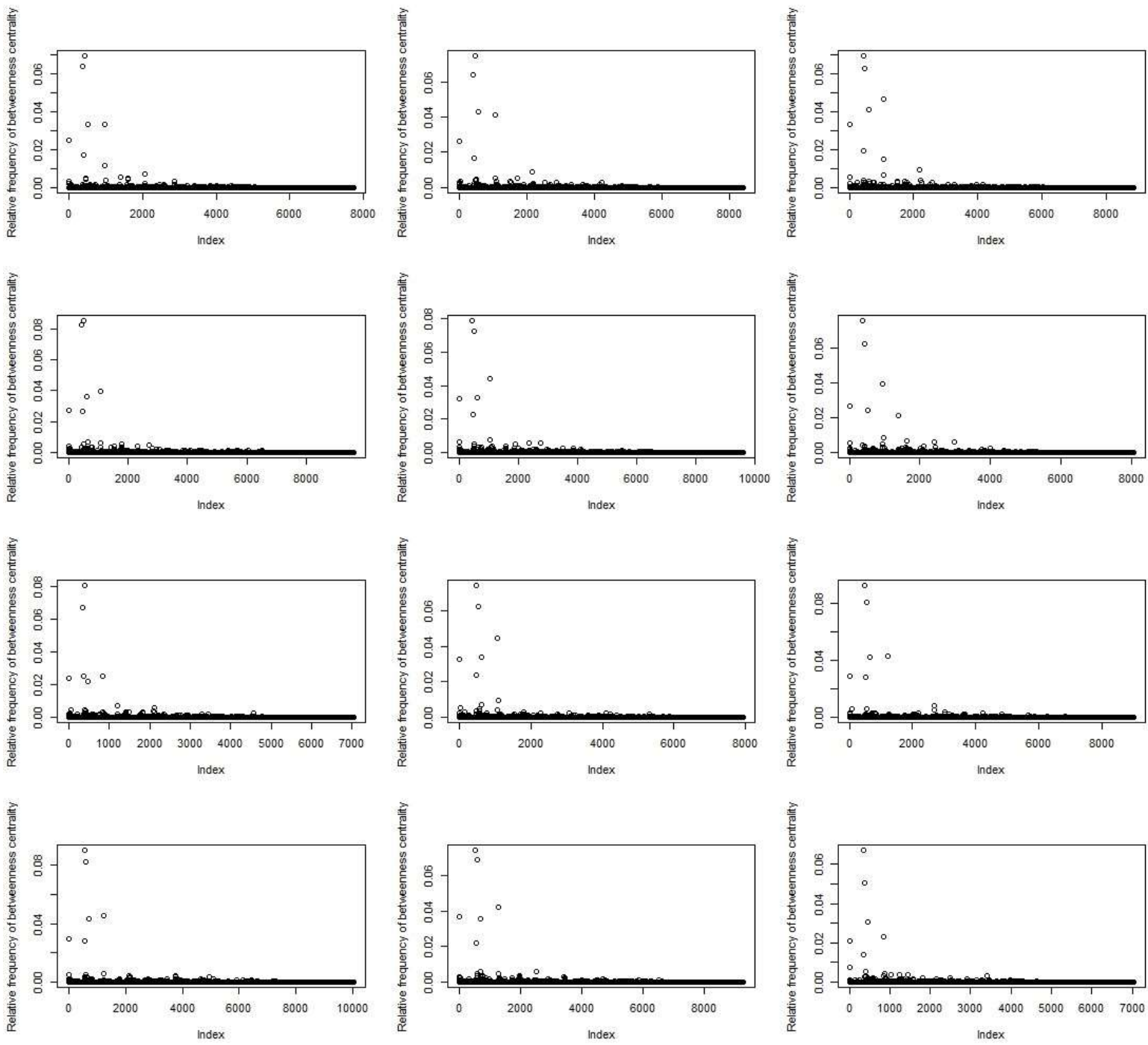

row). 
S23: Normalised betweenness centrality distribution of each network over 2011 (read left to right by
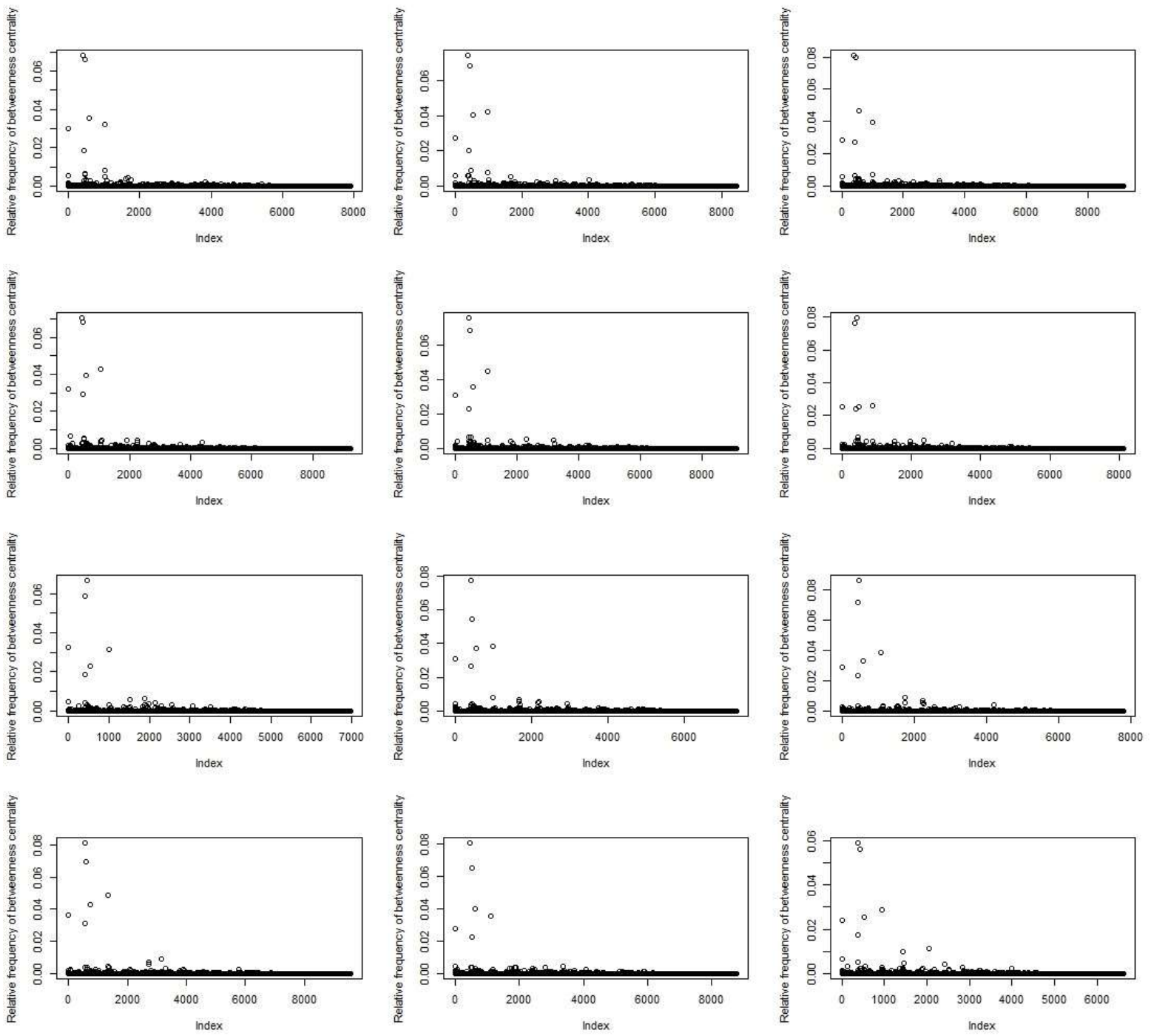

row). 
S24: Normalised betweenness centrality distribution of each network over 2012 (read left to right by
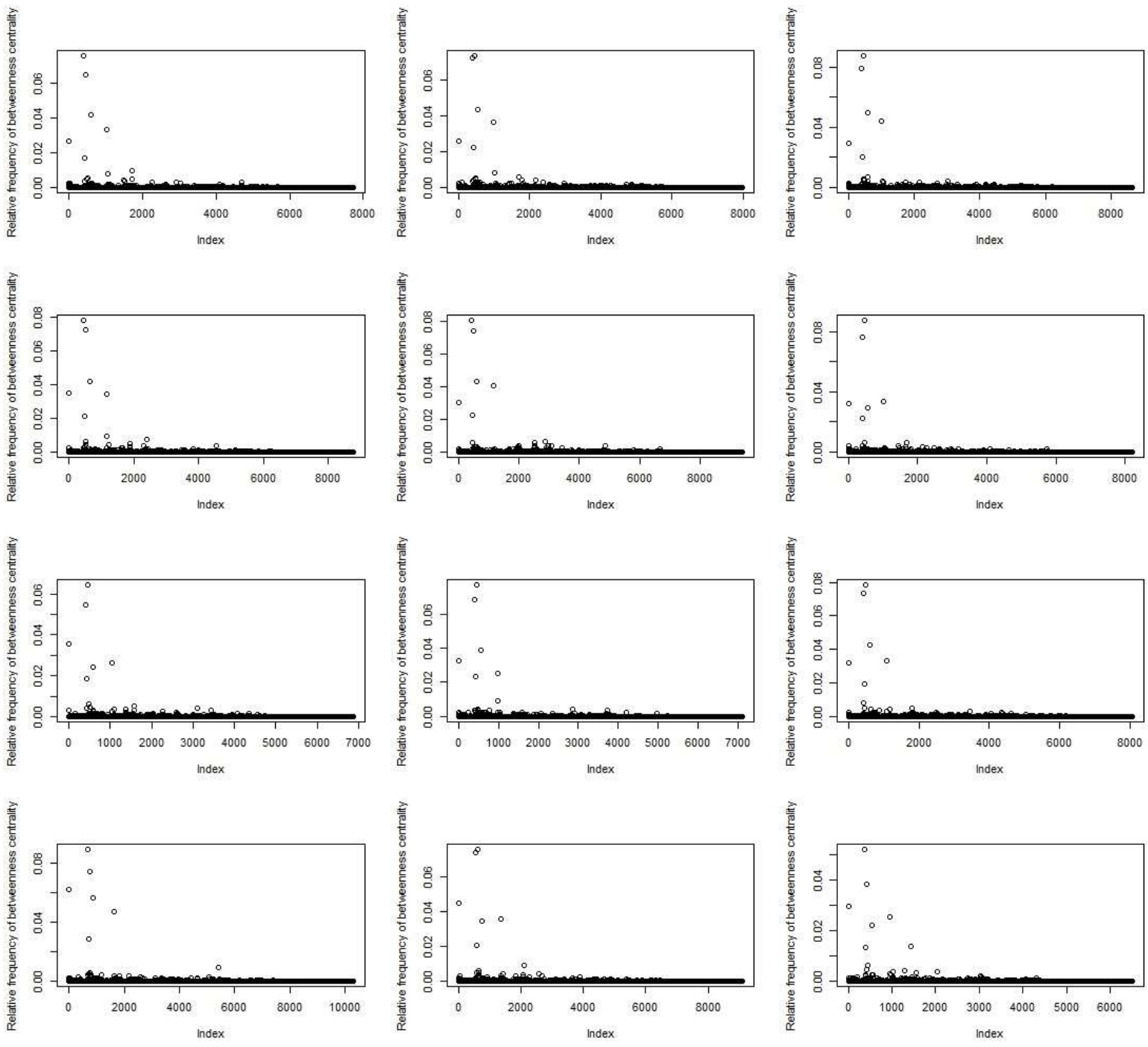

row). 
S25: Normalised betweenness centrality distribution of each network over 2013 (read left to right by
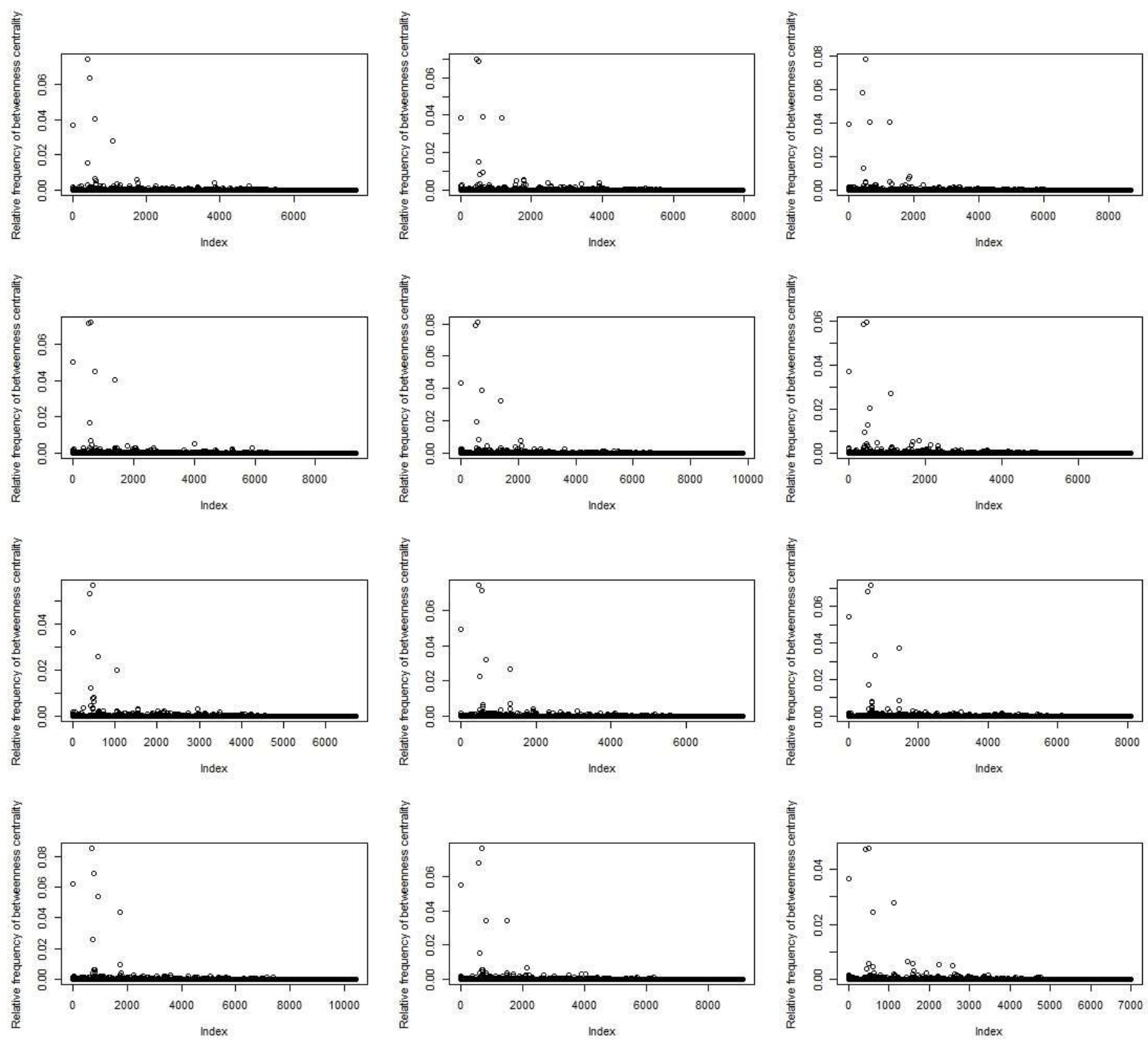

row). 
S26: Normalised betweenness centrality distribution of each network over 2014 (read left to right by
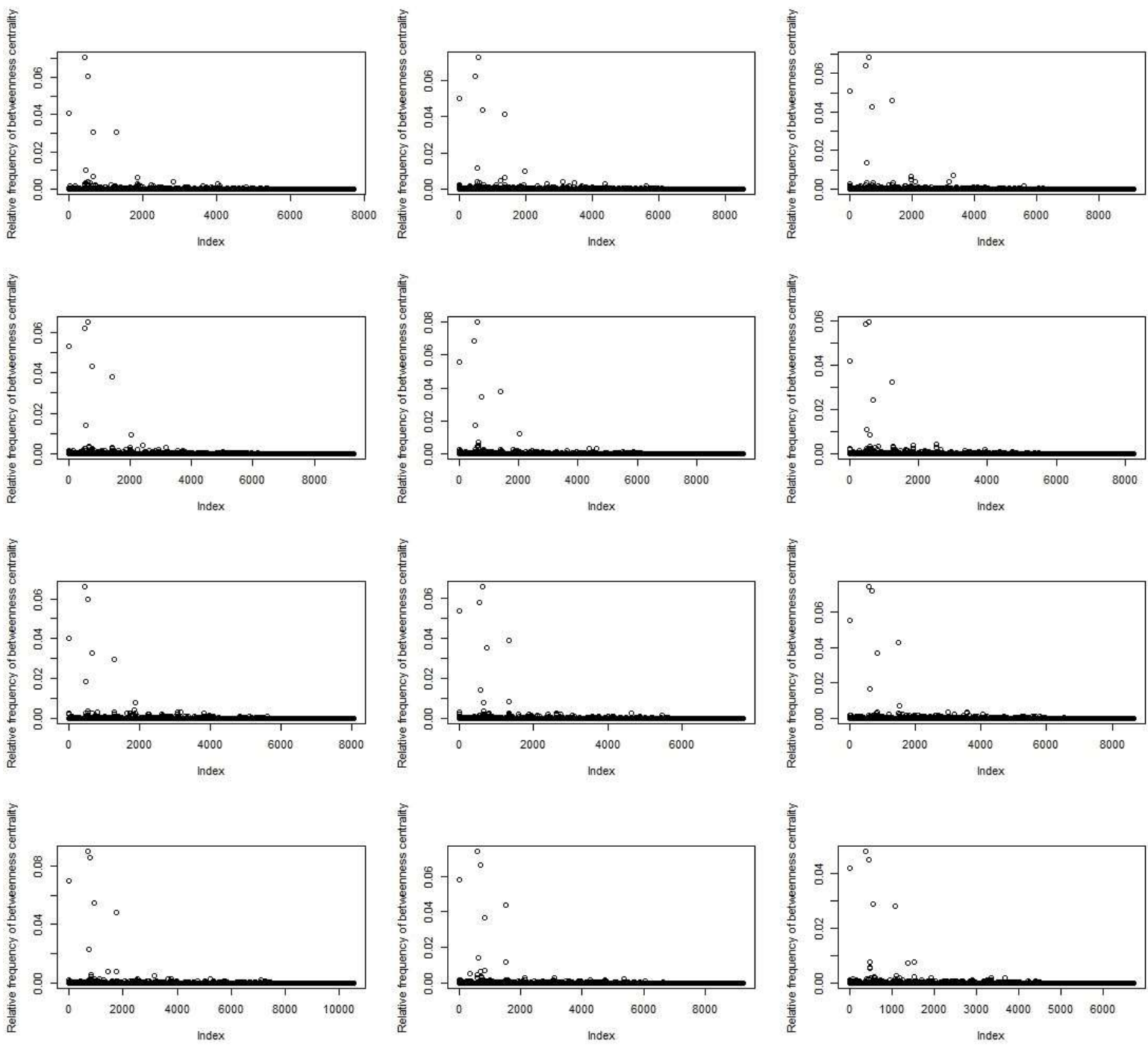

row). 
S27: Normalised betweenness centrality distribution of each network over 2015 (read left to right by

row). 


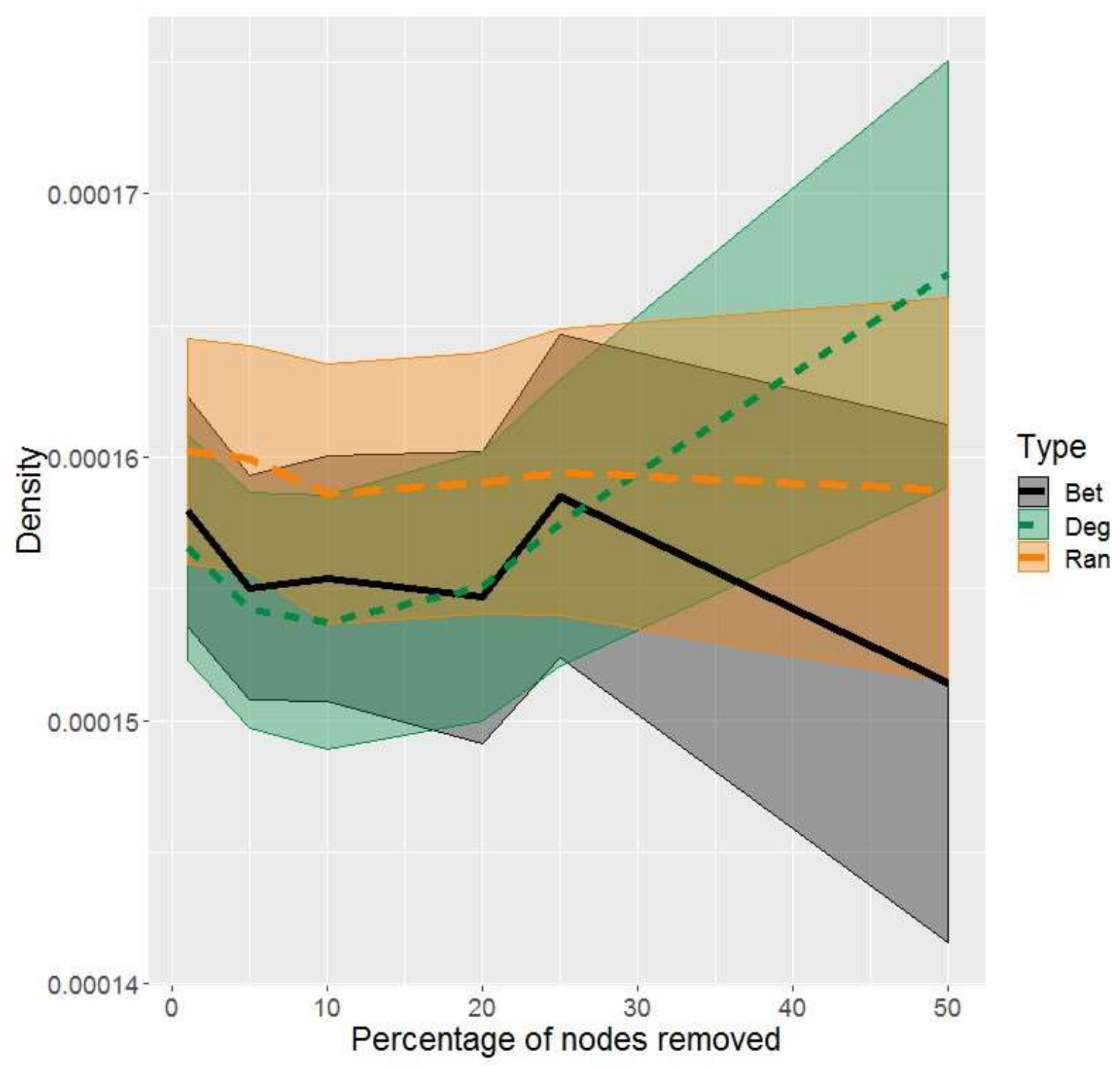

S28: Mean (lines: Black and Solid = Betweenness (Bet) based node removal, Green and Dashed = Degree (Deg) based node removal, Orange and Long-dashed = Random (Ran) node removal) and $95 \% \mathrm{Cl}$ (coloured ribbons: Black $=$ Betweenness based node removal, $\mathrm{Green}=$ Degree based node removal, Orange $=$ Random node removal) density for the networks over 2010-2015 and stratified by node removal. 


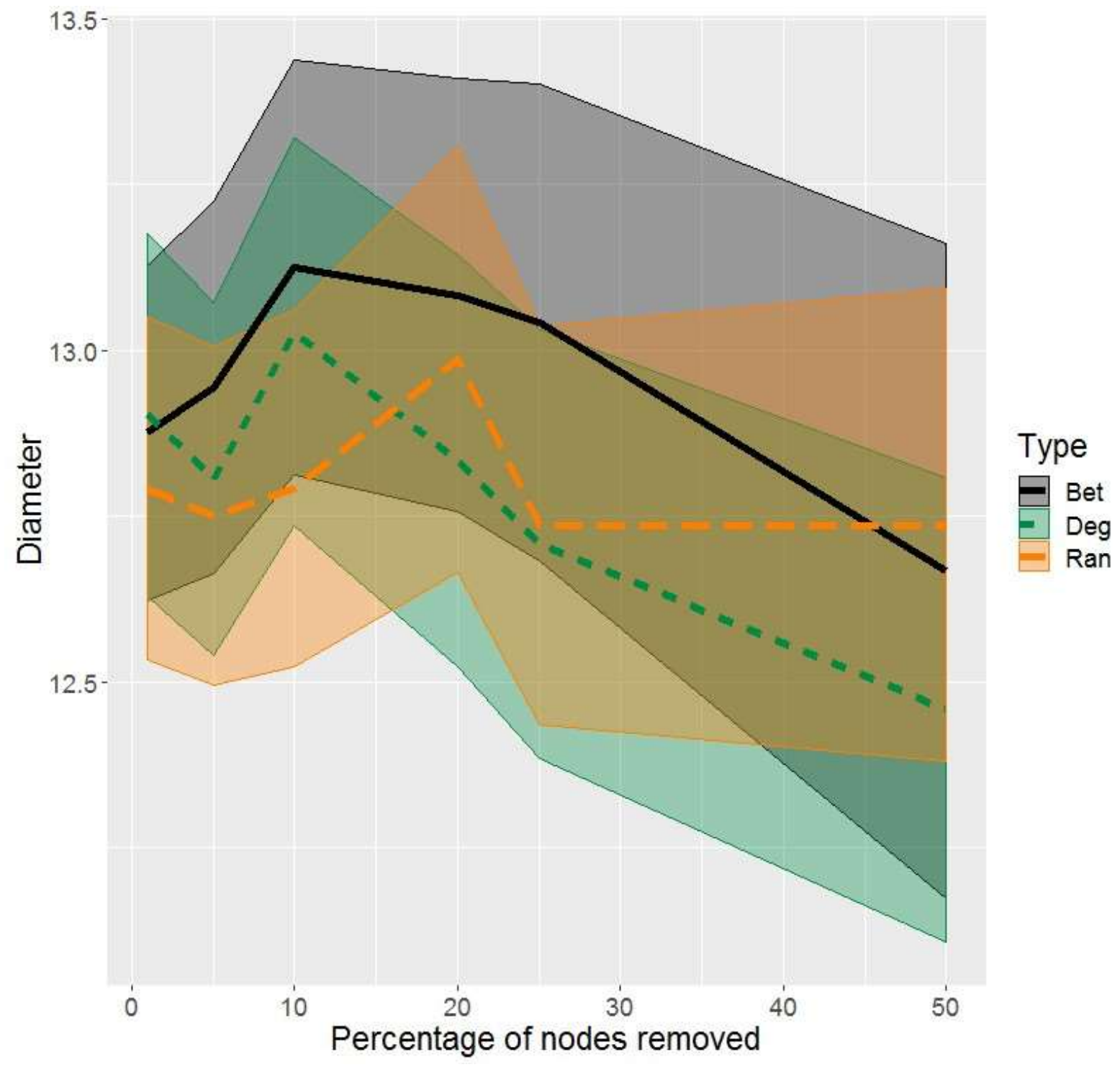

S29: Mean (lines: Black and Solid = Betweenness (Bet) based node removal, Green and Dashed = Degree (Deg) based node removal, Orange and Long-dashed = Random (Ran) node removal) and $95 \% \mathrm{Cl}$ (coloured ribbons: Black = Betweenness based node removal, Green = Degree based node removal, Orange $=$ Random node removal) diameter for the networks over 2010-2015 and stratified by node removal. 


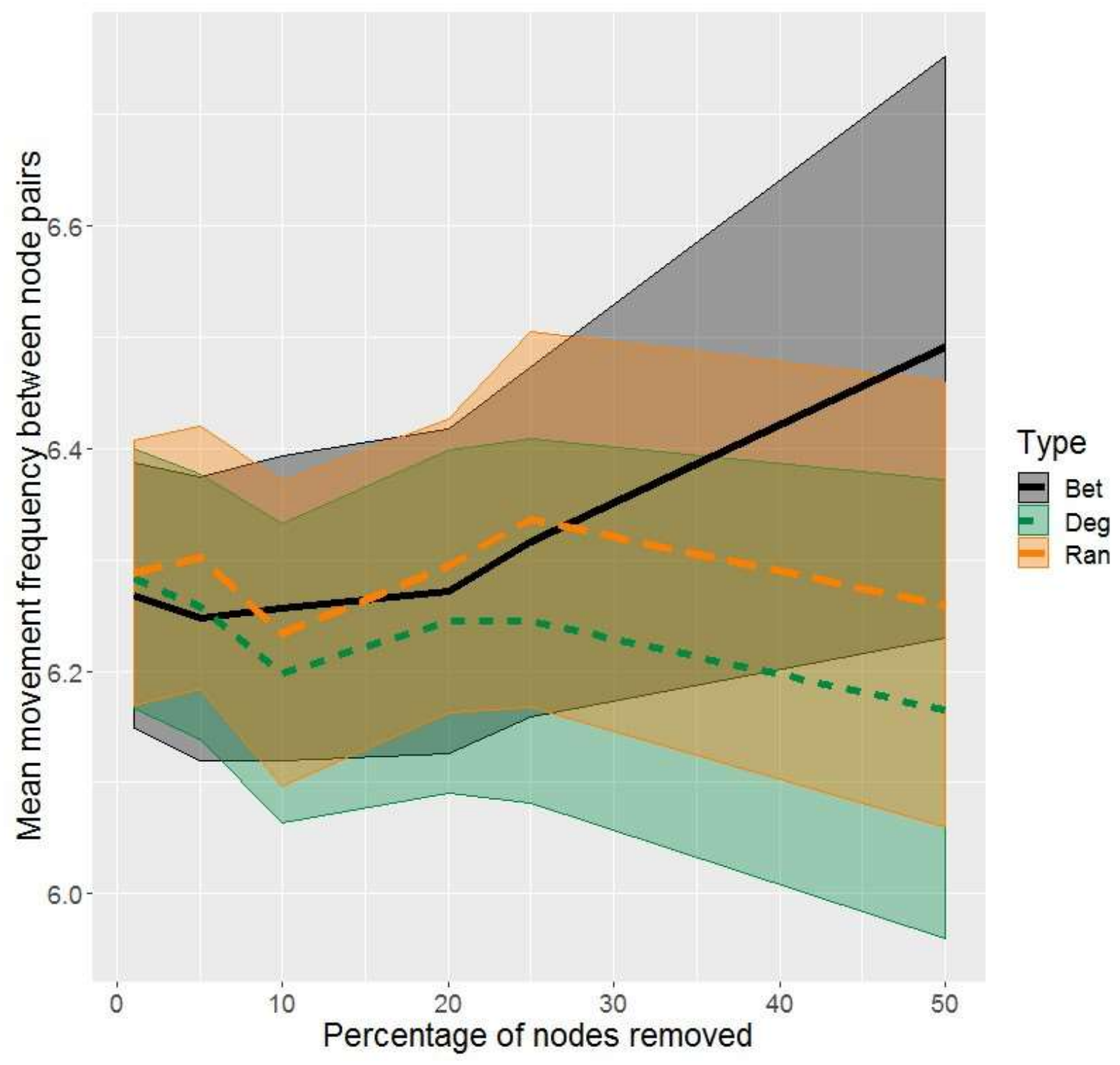

S30: Mean (lines: Black and Solid = Betweenness (Bet) based node removal, Green and Dashed = Degree (Deg) based node removal, Orange and Long-dashed = Random (Ran) node removal) and $95 \% \mathrm{Cl}$ (coloured ribbons: Black = Betweenness based node removal, Green = Degree based node removal, Orange $=$ Random node removal) mean movement frequencies between node pairs for the networks over 2010-2015 and stratified by node removal. 


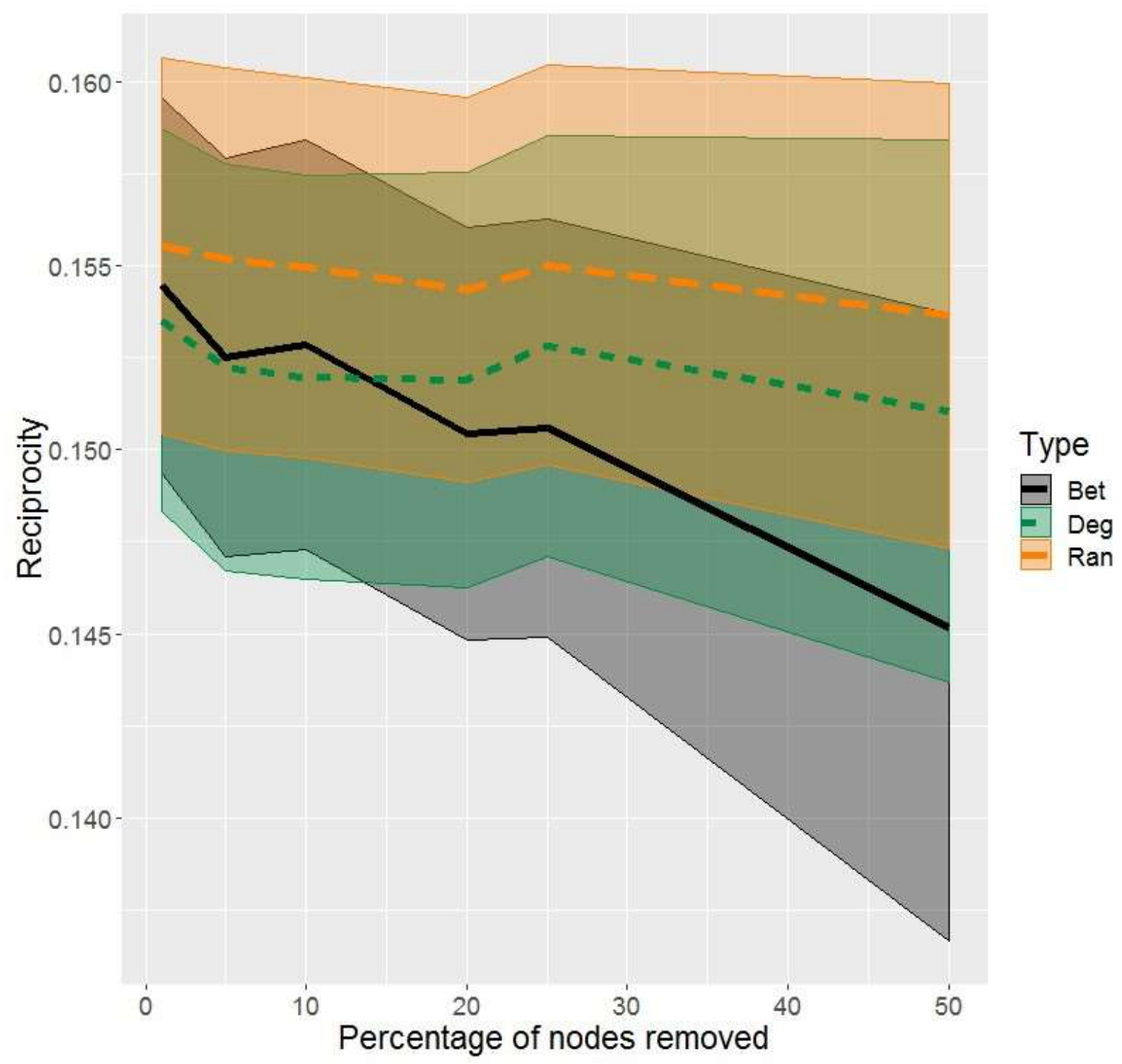

S31: Mean (lines: Black and Solid = Betweenness (Bet) based node removal, Green and Dashed = Degree (Deg) based node removal, Orange and Long-dashed = Random (Ran) node removal) and $95 \% \mathrm{Cl}$ (coloured ribbons: Black $=$ Betweenness based node removal, Green = Degree based node removal, Orange $=$ Random node removal) reciprocity for the networks over 2010-2015 and stratified by node removal. 


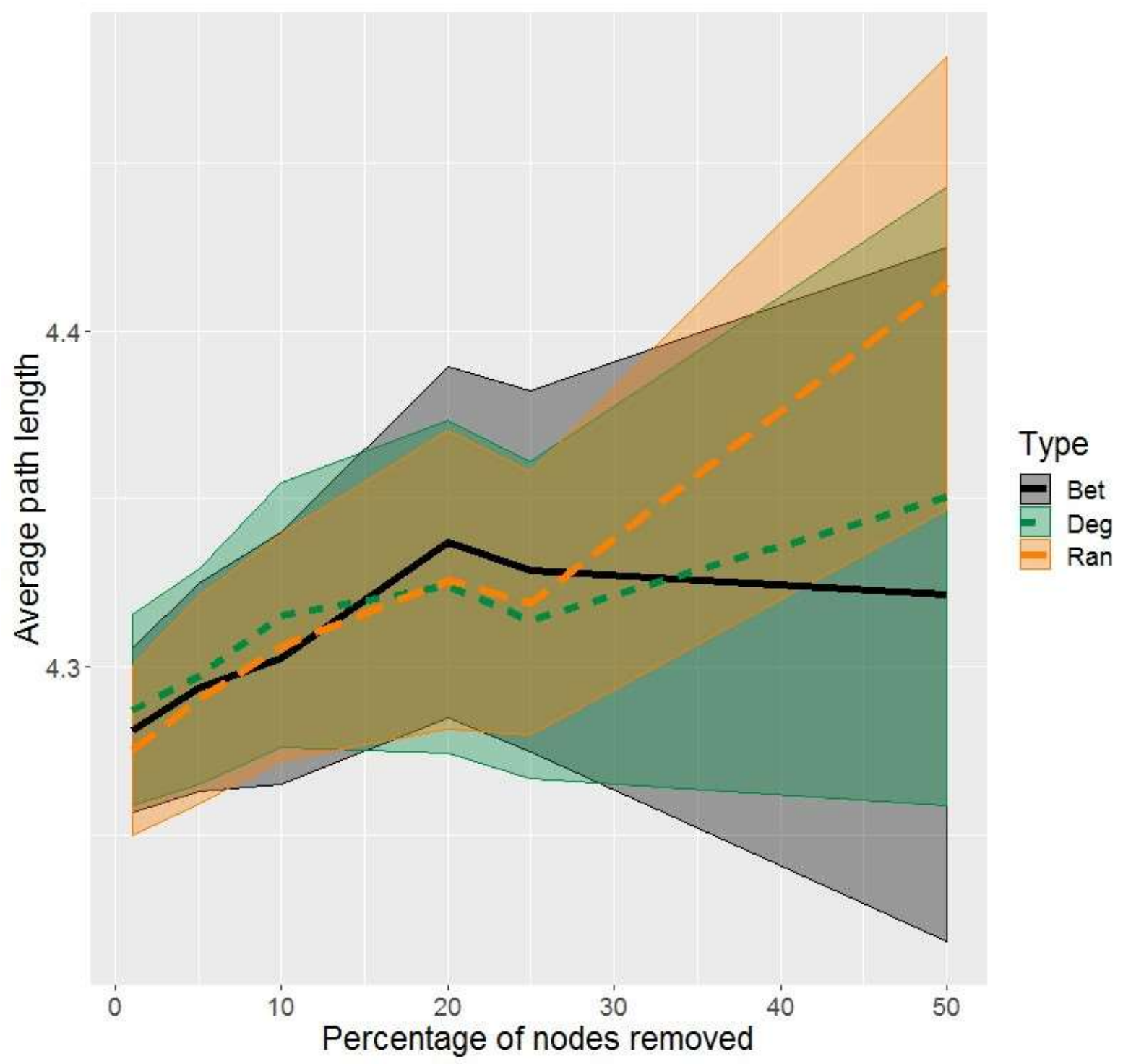

S32: Mean (lines: Black and Solid = Betweenness (Bet) based node removal, Green and Dashed = Degree (Deg) based node removal, Orange and Long-dashed = Random (Ran) node removal) and $95 \% \mathrm{Cl}$ (coloured ribbons: Black $=$ Betweenness based node removal, Green = Degree based node removal, Orange $=$ Random node removal) average path length for the networks over 2010-2015 and stratified by node removal. 